\title{
A Posteriori Bounds for Linear-Functional Outputs of Crouzeix-Raviart Finite Element Discretizations of the Incompressible Stokes Problem*
}

\author{
Marius Paraschivoiu ${ }^{\dagger} \quad$ Anthony T. Patera ${ }^{\ddagger}$
}

March 3, 1998

\begin{abstract}
We present a finite element technique for the efficient generation of lower and upper bounds to outputs which are linear functionals of the solutions to the incompressible Stokes equations in two space dimensions; the finite element discretization is effected by Crouzeix-Raviart elements, the discontinuous pressure approximation of which is central to our approach. The bounds are based upon the construction of an augmented Lagrangian: the objective is a quadratic "energy" reformulation of the desired output; the constraints are the finite element equilibrium equations (including the incompressibility constraint), and the intersubdomain continuity conditions on velocity. Appeal to the dual max-min problem for appropriately chosen candidate Lagrange multipliers then yields inexpensive bounds for the output associated with a fine-mesh discretization; the Lagrange multipliers are generated by exploiting an associated coarse-mesh approximation. In addition to the requisite coarse-mesh calculations, the bound technique requires solution only of local subdomain Stokes problems on the fine-mesh. The method is illustrated for the Stokes equations, in which the outputs of interest are the flowrate past, and the lift force on, a body immersed in a channel.
\end{abstract}

\section{Introduction}

Fast solvers are essential in engineering design due to the large number of appeals to the simulation performed within a design cycle. Indeed, the search for faster solution strategies remains a major research objective. Parallel computing, domain decomposition, preconditioners, higher-order schemes, and adaptive methods are just some of the successful techniques that are being brought to bear on this important problem.

* Submitted to the International Journal for Numerical Methods in Fluids. Address all correspondence to Marius Paraschivoiu, University of Colorado, Department of Computer Science ECOT 722, Campus Box 430, Boulder, CO, 80309, marius@cs.colorado.edu.

${ }^{\dagger}$ Department of Mechanical Engineering, MIT.

${ }^{\ddagger}$ Department of Mechanical Engineering, MIT. 
In actual practice, a typical design effort consists of the optimization of an objective function with respect to selected design variables. The quantity of interest in such objective functions is typically not the entire field solution, but rather a characteristic metric of the system that we will term an "output": for design applications, this output value is more relevant than the entire field solution. Recently, a fast approach has been developed $[19,20$, 21,22 ] to calculate rigorous bounds to an output at a fraction of the cost of a traditional solver.

This technique calculates lower and upper bounds to outputs which are linear functionals of the solution to coercive partial differential equations; recent extension to noncoercive and nonlinear problems is discussed in [25]. The bounds are for the output associated with a very accurate spatial discretization which we shall call the "truth" mesh: direct calculation of the output on this discretization would be extremely expensive. In our approach, the computation of the bounds nevertheless remains inexpensive; consisting of only global solves on a coarse-mesh: domain decomposition performed along the edges of this coarse-mesh; and finally, calculations of local subdomain Neumann problems on the "truth" mesh. In fact the coarse-mesh may be considered as the "working" mesh utilized in a design cycle: the bound values then serve to relate the accuracy of the design optimization to the "truth". The technique is based on the construction of an augmented Lagrangian, in which the objective is a quadratic energy reformulation of the desired output, and the constraints are the finite element equilibrium conditions and inter-subdomain continuity requirements; the bounds are then derived by evoking the dual max-min problem for appropriately chosen candidate Lagrange multipliers.

In this paper we extend this technique to the incompressible Stokes problem [21], of interest in its own right, but also as a precursor to the in.compressible Navier-Stokes equations. The new considerations addressed are threefold. First, the Stokes problem is itself a constrained minimization problem. Therefore our Lagrangian must be modified to include an additional primal variable, the pressure, and an additional Lagrange multiplier to impose the incompressibility constraint. Second, the pressure term contained in our new Lagrangian will not be controlled by the energy term, which may thus lead to infinite bounds. The solution to this difficulty is the use of the Crouzeix-Raviart element [15, 26], which permits us to exactly eliminate the dependence of the Lagrangian on the pressure variable through a projection technique which, thanks to the discontinuous (and hence decoupled) pressure space, can be effected solely through problems local to each element. Third, higher-order velocity approximation is required in the Stokes problem to satisfy the inf-sup condition. This requirement also necessitates higher-order hybrid flux construction, which is developed here in a formulation similar to that described in [5]. Finally, regarding computational savings, the domain decomposition of the Stokes problem offers even more substantial savings than the domain decomposition of elliptic problems.

Our work has benefited from previous efforts in the a posteriori error estimation community $[3,5,7,8,9,10,13,14,17,27,30]$. In earlier papers $[19,20,22]$ we have described the similarities between our bounds technique and both "explicit" and "implicit" a posteriori error estimators for elliptic equations. Similarly to earlier implicit techniques, we base our bounds technique on local independent subproblem calculations. However, our bounds 
offer the advantage of measuring the error in norms different than the energy norm. Indeed, for quantitative confirmation of engineering design quantities, we must measure the error directly in the norm associated with these outputs. Recent explicit error indicators for the error in linear-functional outputs have been developed [14] based on the Aubin-Nitsche duality procedure. These techniques allow adaptive improvement of finite element predictions for the desired engineering output, and can also be applied to the Navier-Stokes equations. However, these estimates are less quantitative than ours due to the presence of constants that cannot be precisely evaluated, and thus the goal of design confirmation is less satisfactory achieved.

For the Stokes problem, Verfürth [31] has developed implicit error estimates based on the solution of local Stokes problems, and explicit estimators based on a suitable evaluation of the residual of the finite element solution, that provide estimates for the error in the energy norm. In [12], Bank and Welfred successfully reconsider the implicit error estimators for the Stokes problem. A comparison of all of these methods [11] indicates that the estimates are a good indicator of the error, that the explicit estimator is about a factor of two less expensive than the implicit estimators, and that the implicit estimators require about one fourth the computing time needed for the solution process. We note that these estimators have been developed for the mini-element discretization of the Stokes problem [2], which is based on piecewise continuous linear velocities augmented with quadratic bubble functions and piecewise continuous linear pressures. Our technique is limited to the discretization of the Stokes problem by Crouzeix-Raviart (discontinuous pressure) elements.

Less standard approaches to measuring the error have been proposed in [18. 4]. The error estimators proposed by Ladeveze et al. [18] measure the error in the constitutive law of materials in the limit of incompressible solids. (Recall that there is a direct analogy between an incompressible linear-elastic isotropic solid in equilibrium and an incompressible Newtonian fluid in the steady creeping limit [23].) Another implicit estimator for the Stokes problem is found in [4]: in this approach the error estimator is based on local residual problems which require only the solution of decoupled subdomain problems of Poisson type with Neumann data. Although this method has the advantage of being faster than implicit methods that require solution of local Stokes problems, the bounds obtained are for an "equivalent" energy norm, and thus not directly relevant to validation and confirmation in engineering design.

We remark that most of the previous work on a posteriori Stokes error analysis is focused on estimating the error for application to mesh adaptivity rather than directly addressing engineering design problems; there is a relative lack of methods for validation and confirmation which focus on rigorously quantifying the error in the outputs of interest. Nevertheless, the utility of adaptive mesh technology indicates that our technique must be extended to quantify the error locally for use in adaptive error control procedures. For elliptic Partial Differential Equations such an extension has already been presented in [24]; generalization to the Stokes problem, though not considered here, should be relatively straightforward.

The outline of the remainder of the paper is as follows. In Section 2 we describe our model problem and the output linear functionals which we will investigate. In Section 3 we present the finite element discretization to which we apply our bounds technique. In Sections 4 and 5 
we describe and prove the bounds procedure and the properties of our estimates. In Section 6 we develop an approach to decrease the bound gap. Finally, in Section 7, we illustrate our technique for the Stokes problem and associated outputs of interest to demonstrate the engineering relevance of our technique.

\section{Model Problem}

\subsection{Governing Equations}

We consider the steady creeping flow of an incompressible ( $\rho=$ constant) Newtonian fluid with constant dynamic viscosity, $\mu$, between two plates with a periodic array of rectangle obstacles in the center. This geometry is presented in Figure 1. where $\left(x_{1}, x_{2}\right)$ denotes the coordinate system with corresponding unit vectors $\mathbf{e}_{1}, \mathbf{e}_{2} ; \Omega$ is the domain; and $\Gamma_{j}, j=$ $1, . ., 5$, are the domain boundary segments. The flow is driven by a forcing term which can be interpreted as a pressure gradient $\Delta P / L$ in the $\mathrm{e}_{1}$ direction. The velocity and pressure perturbations are periodic in the $e_{1}$ direction.

To describe this flow we use the "Laplacian" form of the incompressible Stokes equations. In Gibbsian notation, the velocity vector $u$ and the scalar perturbation pressure field $p$ satisfy

$$
\begin{aligned}
-\Delta \mathbf{u}+\nabla p=\mathbf{f}, & \text { in } \Omega, \\
-\nabla \cdot \mathbf{u}=0, & \text { in } \Omega,
\end{aligned}
$$

with no-slip Dirichlet and periodic boundary conditions,

$$
\begin{aligned}
\mathbf{u} & =0 \text { on } \Gamma_{i}, i \in\{1,3,5\} \\
\left.\mathbf{u}\right|_{\Gamma_{2}} & =\left.\mathbf{u}\right|_{\Gamma_{4}} \\
\left.\nabla \mathbf{u}\right|_{\Gamma_{2}} & =\left.\nabla \mathbf{u}\right|_{\Gamma_{4}} .
\end{aligned}
$$

Here $\mathrm{f}$ is the volumetric force, $\mathrm{f}^{T}=\left[\begin{array}{ll}1 & 0\end{array}\right]$; for convenience we set the viscosity to unity. We also require that $\int_{\Omega} p \mathrm{~d} A=0$ for uniqueness.

The variational form of $(1)-(2)$ is: Given $f \in\left(\mathcal{H}^{-1}(\Omega)\right)^{2}$, find $u=\left(u_{1}, u_{2}\right) \in\left(\mathcal{H}_{0}^{1}(\Omega)\right)^{2}$ and $p \in L_{0}^{2}(\Omega)$ such that

$$
\begin{aligned}
\int_{\Omega} \nabla \mathbf{v} \cdot \nabla \mathbf{u}-p \nabla \cdot \mathbf{v}-\mathbf{v} \cdot \mathbf{f} \mathrm{d} A & =0 \quad \forall \mathbf{v} \in \mathcal{H}_{0}^{1}(\Omega) \otimes \mathcal{H}_{0}^{1}(\Omega), \\
-\int_{\Omega} q \nabla \cdot \mathbf{u} \mathrm{d} A & =0 \quad \forall q \in L_{0}^{2}(\Omega),
\end{aligned}
$$

where $\mathrm{d} A$ is a differential area element, and

$$
\begin{aligned}
\mathcal{H}_{0}^{1}(\Omega) & =\left\{v \in \mathcal{H}^{1}(\Omega)|v|_{\Gamma_{2}}=\left.v\right|_{\Gamma_{4}} ;\left.v\right|_{\Gamma_{1}}=0, i \in\{1,3,5\}\right\} \\
L_{0}^{2}(\Omega) & =\left\{q \in L^{2}(\Omega) \mid \int_{\Omega} q \mathrm{~d} A=0\right\}
\end{aligned}
$$

where $\mathcal{H}^{1}(\Omega)$ and $L^{2}(\Omega)$ are the usual Sobolev spaces [1]. We also introduce $\mathcal{X}=\mathcal{H}^{1}(\Omega) \otimes$ $\mathcal{H}^{1}(\Omega), \mathcal{Y}=L^{2}(\Omega), \mathcal{X}^{0}=\mathcal{H}_{0}^{1}(\Omega) \otimes \mathcal{H}_{0}^{1}(\Omega)$, and $\mathcal{Y}^{0}=L_{0}^{2}(\Omega)$. 


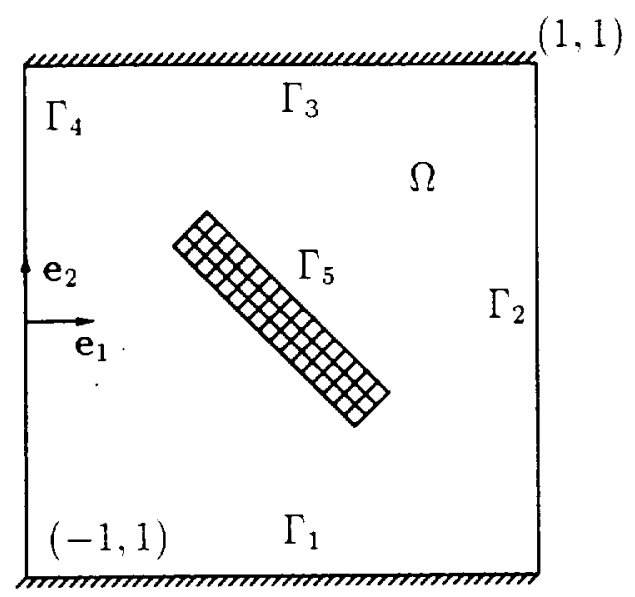

Figure 1: Computational domain: $\Gamma_{1}, \Gamma_{3}$ and $\Gamma_{5}$ are homogeneous Dirichlet boundaries, $\Gamma_{4}$ and $\Gamma_{2}$ are periodic boundaries.

\subsection{Output Linear Functionals}

We assume that our output, $s$, may be expressed as a linear (or more generally, affine) functional of the velocity components $u$, and a linear functional of the pressure $p$, that is $s=\ell(\mathbf{u}, p)=\ell^{l}(\mathbf{u})+\ell^{P}(p)$ where

$$
\ell: \mathcal{X} \odot \mathcal{Y} \rightarrow \mathbf{R}
$$

or

$$
\begin{array}{ll}
\ell^{V} & : \mathcal{X} \rightarrow \mathbf{R} \\
\ell^{P} & : \mathcal{Y} \rightarrow \mathbf{R} .
\end{array}
$$

It is clear that $\ell$ is a linear functional on the product space $\mathcal{X} \times \mathcal{Y}$. On physical grounds, $\ell^{P}(1)=0$, since the pressure level is arbitrary, and thus must not affect the output; the mathematical ramifications of this condition will become clear later.

Examples of possible linear functionals include the flowrate through the channel, or the lift force on the body immersed in the fluid. The particular linear functional for the flowrate (output $s^{(1)}$ ) is defined as

$$
\begin{aligned}
& \ell^{v}(\mathbf{v})=\frac{L}{\Omega} \int_{\Omega} \mathbf{v} \cdot \mathbf{e}_{1} \mathrm{~d} A, \forall \mathbf{v} \in \mathcal{X}, \\
& \ell^{P}(q)=0, \forall q \in \mathcal{Y},
\end{aligned}
$$

where $L(=2)$ is the height between the plates. Note that this output functionals is bounded for all $\mathrm{v}$ in $\mathcal{X}$. Another important engineering output of interest $\left(s^{(2)}\right)$ is the lift force acting on a body. We evaluate this force with the following functionals:

$$
\ell^{v}(\mathrm{v})=\int_{\Omega} \nabla \chi \cdot \nabla \mathrm{v}-\chi \cdot \mathbf{f} \mathrm{d} A
$$




$$
\ell^{P}(q)=-\int_{\Omega} q \nabla \cdot \chi \mathrm{d} A
$$

or equivalently

$$
s^{(2)}=\int_{\Omega} \nabla \chi \cdot \nabla \mathbf{u}-p \nabla \cdot \chi-\chi \cdot \mathrm{f} \mathrm{d} A,
$$

where $\chi$ is any continuous function in $\mathcal{X}$ such that $\chi \cdot \mathbf{e}_{2}=1$ on $\Gamma_{5}$ and $\chi=0$ on the other non-periodic boundaries.

The motivation behind the choice (13) is once again to obtain bounded functionals, since we can easily predict specific convergence properties only for $\ell^{V} \in \mathcal{H}^{-1}(\Omega)$ and $\ell^{P} \in L^{2}(\Omega)$ : it is shown in [21] that (13) is indeed bounded. To show that we correctly reproduce the lift, we first note that it corresponds to

$$
s^{(2)}=\int_{\Omega} \nabla \cdot(\chi \cdot \nabla \mathbf{u})+\nabla \cdot((\chi \cdot \nabla) \mathbf{u})-\nabla \cdot(p \chi) \mathrm{d} A-\int_{\Omega} \nabla \cdot((\chi \cdot \nabla) \mathbf{u}) \mathrm{d} A .
$$

By application of Gauss' theorem we then obtain

$$
s^{(2)}=\int_{\partial \Omega} \chi \cdot(\sigma \hat{\mathbf{n}}) \mathrm{d} s-\int_{\Omega} \nabla \cdot((\chi \cdot \nabla) \mathbf{u}) \mathrm{d} A,
$$

where $\sigma$ is the stress tensor and $\hat{\mathrm{n}}$ is the outward normal vector on the domain boundary. Finally, we demonstrate in [21] that the term $\int_{\Omega} \nabla \cdot((\chi \cdot \nabla) \mathbf{u}) \mathrm{d} A$ is zero for smooth solutions, since both the tangential and normal derivatives of the normal velocity vanish, the latter thanks to incompressibility; from our definition of $(16), s^{(2)}$ thus reduces to the lift. (Note that future work should consider the stress formulation of the Stokes equations, which more naturally generates the stress contributions on the boundary:) The functional, (13), also permits the calculation of the drag force acting on the body similarly by choosing a function $\chi$ such that $\chi \cdot \mathrm{e}_{1}=1$ on $\Gamma_{5}$.

We close this section with two remarks. First, if we choose $\chi$ to be an incompressible field, then lie pressure part of the functional $\left(\ell^{P}(p)\right)$ is zero. To show that this choice is compatible with the boundary condition, we apply Gauss' theorem to find

$$
\int_{\Omega} \nabla \cdot \chi \mathrm{d} A=\int_{\partial \Omega} \chi \cdot \hat{\mathrm{n}} \mathrm{d} s=\int_{\Gamma_{5}} \chi \cdot \hat{\mathrm{n}} \mathrm{d} s=0
$$

where the final equality obtains since $\Gamma_{5}$ is a closed boundary contour and $\chi \cdot \mathbf{e}_{2}=1$ on $\Gamma_{5}$. Second, we note that (17) also proves that, as required, $\ell^{P}(1)=0$ in (13).

\section{Finite Element Discretization}

We first introduce the necessary triangulations, and the general finite element ingredients, such as the bilinear and linear forms and function spaces. that will be required in what follows. 


\subsection{Triangulations}

Two different types of triangulations are required for our "hierarchical" bound procedure, the $H$-mesh and the $h$-mesh, where the latter is a refinement of the former. The $h$-mesh is our fine mesh, which serves as the "truth" mesh; by "truth" we indicate our assumption that the difference between the numerical solution obtained for this fine-mesh and the exact solution is negligible. The $H$-mesh is our "working" mesh, which is used, in conjunction with local Stokes problems, to calculate the bounds.

As our $H$-mesh discretization of $\Omega$ we take a geometrically conforming regular triangulation $\mathcal{T}_{H}$ consisting of $K$ triangles $T_{H}$ such that

$$
\bar{\Omega}=\bigcup_{T_{H} \in \mathcal{T}_{H}} \bar{T}_{H}
$$

We denote the set of all (open) edges $\gamma$ of this triangulation as $\mathcal{E}\left(\mathcal{T}_{H}\right)$, and the set of three edges $\gamma T_{H}$ associated with each element $T_{H}$ as $\mathcal{E}\left(T_{H}\right)$. We denote the set of interior edges as $\mathcal{E}_{\text {int }}\left(\mathcal{T}_{H}\right)$, and the sets of Dirichlet edges - the edges that are part of Dirichlet boundary segments - as $\mathcal{E}_{D}\left(\mathcal{T}_{H}\right)$. We denote the set of $N$ vertices of the triangulation by $\mathcal{M}\left(\mathcal{T}_{H}\right)$.

The triangulation and elemental edges are, of course, related. In particular, given an edge $\gamma T_{H}$ in $\mathcal{E}\left(T_{H}\right)$, we shall indicate the coincident edge $\gamma$ in $\mathcal{E}\left(\mathcal{T}_{H}\right)$ as $\gamma=E\left(\gamma_{T_{H}}\right)$. We next associate with each edge $\gamma$ in $\mathcal{E}\left(\mathcal{T}_{H}\right)$ a unique normal $\hat{\mathbf{n}}^{\gamma}$ such that, if $\gamma$ lies on $\partial \Omega, \hat{\mathbf{n}}^{\gamma}$ coincides with the outward normal $\hat{\mathbf{n}}$ on $\partial \Omega$. Then, for all $T_{H}$ in $\mathcal{T}_{H}$, and all edges $\gamma_{T_{H}}$ in $\mathcal{E}\left(T_{H}\right)$, we define

$$
\sigma_{T_{H}}^{\gamma_{H}}=\hat{\mathbf{n}}^{E\left(\gamma_{H}\right)} \cdot \hat{\mathbf{n}}^{\gamma_{T} T_{H}}
$$

where $\hat{\mathbf{n}}^{\gamma_{T_{H}}}$ is the outward normal on $\gamma_{T_{H}}$ with respect to $T_{H}$. In essence, $\sigma_{T_{H}}^{\gamma_{T_{H}}}$ is \pm 1 on the two "sides" of an edge $\gamma$ in $\mathcal{E}\left(\mathcal{T}_{H}\right)$.

Finally, we introduce the $h$-mesh triangulation $\mathcal{T}_{h}$ consisting of triangles $T_{h}$ such that

$$
\bar{\Omega}=\bigcup_{T_{h} \in T_{h}} \bar{T}_{h}
$$

We shall require that $\mathcal{T}_{h}$ be a refinement of $\mathcal{T}_{H}$, in that we can express each $T_{H}$ in $\mathcal{T}_{H}$ as

$$
\bar{T}_{H}=\bigcup_{T_{h} \in \mathcal{R}_{T_{H}}} \bar{T}_{h}
$$

where $\mathcal{R}_{T_{H}}$ is thus the set of $h$-mesh elements that comprise $T_{H}$. A uniform $R$ refinement will denote an $h$-mesh in which $\mathcal{R}_{T_{H}}$ consists of $R^{2}$ triangles $T_{h}$ similar to $T_{H}$.

\subsection{Bilinear and Linear Forms}

We define here the bilinear and linear forms required for the Stokes problem. We first need to define a "broken" space in which no continuity is required on between elements; this space serves to define functions on the local subdomains. In particular, we define

$$
\mathcal{H}_{*}^{1}(\Omega)=\left\{v \in L^{2}(\Omega)|v|_{T_{H}} \in \mathcal{H}^{1}\left(T_{H}\right), \forall T_{H} \in \mathcal{T}_{H}\right\},
$$


and associated product spaces $\mathcal{X}^{*}=\left(\mathcal{H}_{*}^{1}(\Omega)\right)^{2}, \mathcal{X}_{T_{H}}=\left(\mathcal{H}^{1}\left(T_{H}\right)\right)^{2}$, and $\mathcal{Y}_{T_{H}}=L^{2}\left(T_{H}\right)$.

We now define the bilinear form associated with our operators as

$$
a(w, v)=\sum_{T_{H} \in \mathcal{T}_{H}} a_{T_{H}}\left(\left.w\right|_{T_{H}},\left.v\right|_{T_{H}}\right), \quad \forall(w, v) \in\left(\mathcal{H}_{*}^{1}(\Omega)\right)^{2},
$$

where for all $T_{H}$ in $\mathcal{T}_{H}$

$$
a_{T_{H}}(w, v)=\int_{T_{H}} \nabla w \cdot \nabla v \mathrm{~d} A, \quad \forall(w, v) \in\left(\mathcal{H}^{1}\left(T_{H}\right)\right)^{2}
$$

In addition we denote

$$
a(\mathbf{w}, \mathbf{v})=a\left(w_{1}, v_{1}\right)+a\left(w_{2}, v_{2}\right), \quad \forall(\mathbf{w}, \mathbf{v}) \in\left(\mathcal{X}^{*}\right)^{2}
$$

and

$$
a_{T_{H}}(\mathbf{w}, \mathbf{v})=a_{T_{H}}\left(w_{1}, v_{1}\right)+a_{T_{H}}\left(u_{2}, v_{2}\right) . \quad \forall(\mathbf{w}, \mathbf{v}) \in\left(\mathcal{X}_{T_{H}}\right)^{2}
$$

Similarly,

$$
d(w, q)=\sum_{T_{H} \in \mathcal{T}_{H}} d_{T_{H}}\left(\left.w\right|_{T_{H}},\left.q\right|_{T_{H}}\right), \quad \forall(w, q) \in \mathcal{H}_{*}^{1}(\Omega) \circlearrowright L^{2}(\Omega)
$$

where for all $T_{H}$ in $\mathcal{T}_{H}$

$$
d_{T_{H}}^{i}(w, q)=\int_{T_{H}} q \nabla w \cdot \mathbf{e}_{i} \mathrm{~d} A . \quad \forall(w, q) \in \mathcal{H}^{1}\left(T_{H}\right) \otimes L^{2}(\Omega),
$$

and

$$
d_{T_{H}}(\mathbf{w}, q)=d_{T_{H}}^{1}\left(w_{1}, q\right)+d_{T_{H}}^{2}\left(w_{2}, q\right), \quad \forall(\mathbf{w}, q) \in \mathcal{X}_{T_{H}} \odot \mathcal{Y}_{T_{H}} .
$$

Note that $a$ and $d$ correspond to the Laplacian and divergence operators, respectively.

We next introduce a set of "jump" bilinear and linear forms required in our variational formulation. These forms will be applied in a scalar fashion to each component of the velocity. In particular, we define the bilinear from

$$
b(w, t)=\left.\left.\sum_{T_{H} \in \mathcal{T}_{H}} \sum_{\gamma_{T_{H}} \in \mathcal{E}\left(T_{H}\right)} \sigma_{T_{H}}^{\gamma_{T_{H}}} \int_{\gamma_{T_{H}}} w\right|_{T_{H}} t\right|_{E\left(\gamma_{T_{H}}\right)} \mathrm{d} s, \quad \forall(w, t) \in \mathcal{H}_{*}^{1}(\Omega) \times \mathcal{Q},
$$

and

$$
b(\mathbf{w}, \mathbf{t})=b\left(w_{1}, t_{1}\right)+b\left(w_{2}, t_{2}\right), \forall(\mathbf{w}, \mathbf{t}) \in \mathcal{X}^{*} \times \mathcal{Q}^{2},
$$

where $\left.w\right|_{T_{H}}$ in $(30)$ is to be interpreted as the trace of $\left.w\right|_{T_{H}}$ on $\gamma_{T_{H}}$, and $\mathcal{Q} \equiv \mathcal{H}^{-1 / 2}\left(\mathcal{E}\left(\mathcal{T}_{H}\right)\right)$; note that $t$ is defined only over the edges of the triangulation. Effectively, (30) computes the moments of the jumps in $w$ over internal edges, and the moments of $w$ over boundary edges.

We now introduce our linear functionals. Associated with the volumetric inhomogeneities we have

$$
\ell^{N}(\mathbf{w})=\sum_{T_{H} \in \mathcal{T}_{H}} \ell_{T_{H}}^{N}\left(\left.\mathbf{w}\right|_{T_{H}}\right), \quad \forall \mathbf{w} \in \mathcal{X}^{*}
$$

where for all $T_{H}$ in $\mathcal{T}_{H}$

$$
\ell_{T_{H}}^{N}(\mathbf{w})=\int_{T_{H}} \mathbf{w} \cdot \mathbf{f} \mathrm{d} A, \quad \forall \mathbf{w} \in \mathcal{X}_{T_{H}}
$$


Associated with our output functional, we introduce

$$
\ell^{O^{\prime \prime}}(\mathbf{w})=\sum_{T_{H} \in \mathcal{T}_{H}} \ell_{T_{H}}^{O^{\prime \prime}}\left(\left.\mathbf{w}\right|_{T_{H}}\right), \quad \forall \mathbf{w} \in \mathcal{X}^{*}
$$

such that

$$
\ell^{O^{v}}(\mathbf{w})=\ell^{V}(\mathbf{w}), \quad \forall \mathbf{w} \in \mathcal{X}
$$

Similarly we can introduce a linear functional for the pressure,

$$
\ell^{O^{p}}(q)=\sum_{T_{H} \in \mathcal{T}_{H}} \ell^{O^{p}}\left(q \mid T_{H}\right), \quad \forall q \in \mathcal{Y}^{\circ}
$$

such that

$$
\ell^{O^{p}}(q)=\ell^{P}(q), \quad \forall q \in \mathcal{Y}^{0} .
$$

Here $\ell^{V}()$ and $\ell^{P}()$ are the formal output functionals introduced in (11).

\subsection{Function Spaces}

As already indicated, we consider two different spatial discretizations: $\delta=H$ and $\delta=$ $h$, which correspond to our "working" and "truth" discretizations, respectively. For the Crouzeix-Raviart approximation spaces of interest $[16,26]$ the velocity space is given by

$$
X_{\delta}=\left\{\left.\mathbf{v}\right|_{T_{\delta}} \in\left(\mathbf{P}_{2}^{+}\left(T_{S}\right)\right)^{2}, \forall T_{\delta} \in \mathcal{T}_{\delta}\right\} \bigcap \mathcal{X}^{0}
$$

where $\mathbf{P}_{2}^{+}\left(T_{\delta}\right)=\left\{\mathbf{P}_{2}\left(T_{\delta}\right)+\alpha_{T_{H}} \mathbf{P}_{b}, \alpha_{T_{H}} \in \mathbf{R}\right\}$ is the space of quadratic polynomials enhanced by a cubic "bubble" function $\mathbf{P}_{b}$ over $T_{\varepsilon}$ : for the pressure, we identify

$$
Y_{\delta}=\left\{\left.q\right|_{T_{\delta}} \in \mathrm{P}_{1}\left(T_{\delta}\right), \forall T_{\delta} \in \mathcal{T}_{\delta}\right\} \bigcap \mathcal{Y}^{\circ}
$$

We also introduce spaces of polynomial functions defined on the edges only,

$$
\mathcal{Q}_{k}=\left\{\left.t\right|_{\delta} \in \mathbf{P}_{k}(\gamma), \forall \gamma \in \mathcal{E}\left(\mathcal{T}_{H}\right)\right\} \bigcap \mathcal{Q}
$$

where $k$ identifies the order of the polynomial over the edge $\gamma$.

We now define two subdomain local spaces. First, for the velocity, the working and truth subdomain local spaces are given by

$$
U_{H}\left(T_{H}\right)=\left(\mathbf{P}_{2}^{+}\left(T_{H}\right)\right)^{2}
$$

and

$$
U_{h}\left(T_{H}\right)=\left\{\left.\mathbf{v}\right|_{T_{h}} \in\left(\mathbf{P}_{2}^{+}\left(T_{h}\right)\right)^{2}, \forall T_{h} \in \mathcal{R}_{T_{H}}\right\} \bigcap \mathcal{X}_{T_{H}},
$$

respectively, where we recall that $\mathcal{R}_{T_{H}}$ is the set of $h$-mesh elements that constitute $T_{H}$. We also define corresponding spaces which now include the incompressibility constraint, and define the spaces

$$
Z_{\delta}\left(T_{H}\right)=\left\{\mathrm{v} \in U_{\delta}\left(T_{H}\right)\left|d_{T_{H}}(\mathrm{v}, q)=0\right| \forall q \in M_{\delta}\left(T_{H}\right)\right\}
$$


where

$$
M_{H}\left(T_{H}\right)=\mathbf{P}_{1}\left(T_{H},\right.
$$

and

$$
M_{h}\left(T_{H}\right)=\left\{\left.q\right|_{T_{H}} \in \mathrm{P}_{1}\left(T_{h}\right), \forall T_{h} \in \mathcal{R}_{T_{H}}\right\},
$$

are the local pressure spaces.

Finally, we can define the associated global spaces with and without incompressibility constraint as

$$
V_{\delta}=\left\{\mathrm{v} \in \mathcal{X}^{*}|\mathbf{v}|_{T_{H}} \in U_{\delta}\left(T_{H}\right)\right\}
$$

and

$$
W_{\delta}=\left\{\mathrm{v} \in \mathcal{X}^{*}|\mathrm{v}|_{T_{H}} \in Z_{\delta}\left(T_{H}\right), \forall T_{H} \in \mathcal{T}_{H}\right\},
$$

for $\delta=H$ and $\delta=h$. In essence, the $U_{\delta}\left(T_{H}\right)$ and $Z_{\delta}\left(T_{H}\right)$ are Neumann spaces over each $T_{H}$, for which $V_{\delta}$ and $W_{s}$ are the corresponding global representations. Note that $Z_{\delta}\left(T_{H}\right)$ imposes the necessary "global" incompressibility constraint on the relocity thanks to the discontinuous pressure approximation.

\section{Bound Procedure}

In this Section we present the hierarchical procedure to calculate the bounds. The three principal steps in this procedure are: calculation of the adjoint on the $H$-mesh; calculation of the hybrid flux on the $H$-mesh; and local Stokes solves to obtain the bounds.

\subsection{The $H$-Mesh Adjoint Calculation}

First, we solve the Stokes problem (1)-(2) on the working mesh. We look for $\left(\mathbf{u}_{H}, p_{H}\right) \in$ $X_{H} \times Y_{H}$ such that

$$
\begin{aligned}
a\left(\mathbf{w}, \mathbf{u}_{H}\right)-d\left(\mathbf{w}, p_{H}\right) & =\ell^{N}(\mathbf{w}), \quad \forall \mathbf{w} \in X_{H}, \\
-d\left(\mathbf{u}_{H}, q\right) & =0, \quad \forall q \in Y_{H} .
\end{aligned}
$$

Second, we solve for the output adjoint. We look for $\left(\hat{\boldsymbol{\psi}}_{H}^{ \pm}, \Lambda_{H}^{ \pm}\right) \in X_{H} \times Y_{H}$ such that

$$
\begin{aligned}
a\left(\hat{\psi}_{H}^{ \pm}, \mathbf{w}\right)-d\left(\mathbf{w}, \Lambda_{H}^{ \pm}\right) & =-\left( \pm \ell^{O^{v}}(\mathbf{w})+2 a\left(\mathbf{w}, \mathbf{u}_{H}\right)-\ell^{N}(\mathbf{w})\right), \\
-d\left(\hat{\psi}_{H}^{ \pm}, q\right) & =-\left( \pm \ell^{O^{p}}(q)\right), \quad \forall(\mathbf{w}, q) \in X_{H} \times Y_{H} .
\end{aligned}
$$

Note that because we require $\ell^{P}(1)=0$ we can consider the zero-average space $Y_{H}$, since solvability is ensured. Equivalently, $(51)$ is in fact satisfied over the larger space in which $\mathcal{Y}^{\circ}$ in (39) is replaced by $\mathcal{Y}$. Regarding computational cost, we remark that $(50)$-(51) needs to be solved twice, once for each bound: \pm refers to the pair of solutions required for the lower $(+)$ and upper (-) bounds. If a direct solver is used, only one $\mathrm{LU}$ factorization is required for (48)-(51) - the Stokes operator is, in fact, the same, and only the right-hand side of the equations changes. 
We now define a linear functional $F^{ \pm}(\mathrm{v} ; \mathcal{F}, \mathcal{P})$ which represents the forcing term and the pressure term in the stress balance equations of (50); this functiona? is introduced mainly to simplify the notation. In particular, for any function $\mathcal{F}$ in $\mathcal{X}^{*}$ and $\mathcal{P}$ in $\mathcal{Y}^{\circ}$, we write

$$
F^{ \pm}(\mathrm{v} ; \mathcal{F}, \mathcal{P})=\sum_{T_{H} \in \mathcal{T}_{H}} F_{T_{H}}^{ \pm}\left(\left.\mathbf{v}\right|_{T_{H}} ; \mathcal{F}, \mathcal{P}\right), \quad \forall \mathrm{v} \in \mathcal{X}^{*},
$$

where for all $T_{H}$ in $\mathcal{T}_{H}$,

$$
F_{T_{H}}^{ \pm}\left(\left.\mathbf{v}\right|_{T_{H}} ; \mathcal{F}, \mathcal{P}\right)= \pm \ell_{T_{H}}^{O^{\prime \prime}}(\mathbf{v})+a_{T_{H}}\left(\left.\mathcal{F}\right|_{T_{H}}, \mathbf{v}\right)-\ell_{T_{H}}^{N}(\mathbf{v})-d_{T_{H}}(\mathbf{v}, \mathcal{P})
$$

We can now view the stress balance equations of $(50)$ as

$$
2 a\left(\mathbf{w}, \mathbf{u}_{H}\right)=-F^{ \pm}\left(\mathbf{w} ; \hat{\boldsymbol{\psi}}_{H}^{ \pm}, \Lambda_{H}^{ \pm}\right) .
$$

We can also introduce a second fashion in which to re-express (54), that is

$$
B^{ \pm}\left(\mathbf{v}, \mathbf{u}_{H}\right)=0, \forall \mathbf{v} \in X_{H} .
$$

Here, for any function $\mathcal{G}$ in $\mathcal{X}^{*}$,

$$
B^{ \pm}(\mathbf{v}, \mathcal{G})=\sum_{T_{H} \in T_{H}} B_{T_{H}}^{ \pm}\left(\left.\mathbf{v}\right|_{T_{H}}, \mathcal{G}\right) . \quad \forall \mathbf{v} \in \mathcal{X}^{\prime *},
$$

where for all $T_{H}$ in $\mathcal{T}_{H}$,

$$
B_{T_{H}}^{ \pm}(\mathbf{w}, \mathcal{G})=2 a_{T_{H}}\left(\mathbf{w},\left.\mathcal{G}\right|_{T_{H}}\right)+F_{T_{H}}^{ \pm}\left(\mathbf{w} ; \hat{\psi}_{H}^{ \pm}, \Lambda_{H}^{ \pm}\right), \quad \forall \mathbf{w} \in \mathcal{X}_{T_{H}}^{\prime} .
$$

\subsection{The $H$-Mesh Hybrid Flux Calculation}

The hybrid flux will appear in our Lagrangian as a Lagrange multiplier that enforces the subdomain continuity constraints. Recall that, for the Crouzeix-Raviart elements, we only need to impose continuity for the velocity components. Our procedure here is to calculate the hybrid flux by appealing to the broken space. To start, we have

$$
b\left(\mathbf{v}, \mathbf{y}^{ \pm}\right)=B^{ \pm}\left(\mathbf{v}, \mathbf{u}_{H}\right), \quad \forall \mathbf{v} \in V_{H},
$$

that is, for all $T_{H}$ in $\mathcal{T}_{H}$,

$$
\left.\sum_{\gamma_{T_{H}} \in \mathcal{E}\left(T_{H}\right)} \sigma_{T_{H}}^{\gamma_{T_{H}}} \int_{\gamma_{T_{H}}} \mathbf{v} \cdot \mathbf{y}^{ \pm}\right|_{E\left(\gamma_{T_{H}}\right)} \mathrm{d} s=B_{T_{H}}^{ \pm}\left(\mathbf{v}, \mathbf{u}_{H}\right), \quad \forall \mathbf{v} \in U_{H}\left(T_{H}\right) .
$$

In [21] we present two different approaches to approximate the hybrid flux for quadratic elements based on earlier work for energy-norm estimators $[17,5]$. These techniques are based on an initial approximation which is then corrected with a $\mathbf{P}_{1}$ term to ensure solvability. In addition, a higher-order (quadratic) term is included to improve accuracy; the latter is not required. but should give sharper bounds. We describe here only the most promising 
approach of the two, in which the initial approximation is a $\mathbf{P}_{0}$ approximation. For reasons of simplicity we will present the lower $(+)$ bound hybrid flux calculation; the upper bound proceeds in a similar fashion.

We first introduce $\overline{\mathbf{y}}_{\gamma}^{+} \in\left(\mathcal{Q}_{0}\right)^{2}, \hat{\mathrm{y}}_{\gamma}^{+} \in\left(\mathcal{Q}_{1}\right)^{2}$ and $\tilde{\mathrm{y}}_{\gamma}^{+} \in\left(\mathcal{Q}_{2}\right)^{2}$, which are different polynomial edge functions used in the hybrid flux approximation, $\mathrm{y}^{ \pm}=\overline{\mathbf{y}}_{\gamma}^{ \pm}+\hat{\mathbf{y}}_{\gamma}^{ \pm}+\tilde{\mathbf{y}}_{\gamma}^{ \pm}$. The constant contribution to the hybrid flux, $\overline{\mathbf{y}}_{\gamma}^{+}$, does not present any new subtleties; it is obtained as in [20]. The linear correction, $\hat{\mathbf{y}}_{\gamma}^{+}$, is defined for each component by

$$
\left.\hat{y}_{\gamma}^{+}\right|_{\gamma}=\alpha_{n}^{\gamma} \theta_{n}^{\gamma}(x)+\alpha_{m}^{\gamma} \theta_{m}^{\gamma}(x),
$$

where $\alpha_{n}^{\gamma}$ and $\alpha_{m}^{\gamma}$ are real coefficients to be determined, and $\left(\theta_{n}^{\gamma}(x), \theta_{m}^{\gamma}(x)\right)$ are linear edge functions constructed to be orthogonal to $\left(\left.\varphi_{T_{H}}^{n}\right|_{\gamma},\left.\varphi_{T_{H}}^{m}\right|_{\gamma}\right)[17,10]$. The function $\varphi_{T_{H}}^{n}$ is the restriction of the linear basis function associated with vertex $n$ of $\mathcal{T}_{H}$ to element $T_{H}$. For the quadratic approximation of the hybrid flux we define

$$
\tilde{y}_{\gamma}^{+}=\beta_{\gamma} \rho_{\gamma} \text {. }
$$

where $\rho_{\gamma}: \gamma \rightarrow \mathbf{R}$ is the quadratic function uniquely defined by the conditions:

$$
\int_{\lambda} \rho_{\gamma} \varphi_{T_{H}}^{n} \mathrm{~d} s=0
$$

and

$$
\int_{\gamma} \rho_{\gamma}^{2} \mathrm{~d} s=|\gamma|
$$

where $n$ in (62) refers to either of the two vertices of $\gamma$. We also introduce $\dot{\varphi}_{T_{H}}^{p}$ such that the function $\varphi_{T_{H}}^{n}$ and $\tilde{\varphi}_{T_{H}}^{p}$ associated with $T_{H}$ span $\mathrm{P}_{2}\left(T_{H}\right)$.

Given $\tilde{y}_{\gamma}^{+}$, we calculate the coefficients $\alpha_{n}^{\gamma}$ and thereafter $\hat{y}_{\gamma}^{+}$following the procedure in [20]. To wit, we solve

$$
\sigma_{T_{H}}^{\gamma_{T_{H}}} \int_{\gamma} \varphi_{T_{H}}^{n}\left(\bar{y}_{\gamma}^{+}+\hat{y}_{\gamma}^{+}+\tilde{y}_{\gamma}^{+}\right) \mathrm{d} s=B_{T_{H}}^{+}\left(\varphi_{T_{H}}^{n}, u_{H}\right)
$$

in which we exploit the fact that

$$
\int_{\gamma} \varphi_{T_{H}}^{n}\left(\bar{y}_{\gamma}^{+}+\hat{y}_{\gamma}^{+}+\tilde{y}_{\gamma}^{+}\right) \mathrm{d} s=\int_{\gamma} \varphi_{T_{H}}^{n}\left(\bar{y}_{\gamma}^{+}+\alpha_{n}^{\gamma} \theta_{n}^{\gamma}(x)+\alpha_{m}^{\gamma} \theta_{m}^{\gamma}(x)+\beta_{\gamma}, \rho_{\gamma}\right) \mathrm{d} s=\left(\frac{1}{2} \bar{y}_{\gamma}^{+}+\alpha_{n}^{\gamma}\right)|\gamma|,
$$

since the quadratic function $\rho_{\gamma}$ is orthogonal to the linear functions $\varphi_{T_{H}}^{n}$. To calculate $\tilde{y}_{\gamma}^{+}$ we then follow the procedure in [5], that is, we solve

$$
\sigma_{T_{H}}^{\gamma_{T_{H}}} \int_{\gamma} \tilde{\varphi}_{T_{H}}^{p}\left(\bar{y}_{\gamma}^{+}+\hat{y}_{\gamma}^{+}+\tilde{y}_{\gamma}^{+}\right) \mathrm{d} s=B_{T_{H}}^{+}\left(\tilde{\varphi}_{T_{H}}^{p}, u_{H}\right)
$$

where $\bar{y}_{\gamma}^{+}$and $\hat{y}_{\gamma}^{+}$are now known. Details, in particular as regards solvability, may be found in $[21]$.

To summarize, we first evaluate the non-conforming approximation, $\bar{y}_{\gamma}^{+}$, to the hybrid flux, as in [20]. However, this approximation does not lead to solvability of the equilibrium equation (58). To ensure solvability, we solve $N$ local systems, (64) one for each node of $\mathcal{T}_{H}$, to determine the $\alpha_{n}^{\gamma}$ constants of the linear contribution to $\hat{y}_{\gamma}^{+},(60)$. Finally, we look for a quadratic contribution which leads to (66). Alternative approaches are described in [21]. 


\subsection{The $h$-Mesh Subdomain Neumann Problem}

Before we solve the subdomain problem, we must compute an adjoint $\hat{\psi}_{h}^{ \pm}$on the $h$-mesh. For sharp bounds, $\hat{\psi}_{h}^{ \pm}$should be close to $\hat{\psi}_{H}^{ \pm}$. In addition, the adjoint $\hat{\psi}_{h}^{ \pm}$must be continuous for all $T_{H}$ in $\mathcal{T}_{H}$ to be a valid Lagrange multiplier. Finally, $\hat{\psi}_{h}^{ \pm}$must satisfy an equilibration equation if we are to obtain meaningful bounds - as we will discuss below. Therefore, for all $T_{H}$ in $\mathcal{T}_{H}$, we look for $\hat{\psi}_{h}^{ \pm} \in U_{h}^{D}\left(T_{H}\right)$, such that

$$
\begin{array}{rlrl}
a_{T_{H}}\left(\mathbf{v}, \hat{\psi}_{h}^{ \pm}-\hat{\psi}_{H}^{ \pm}\right)-d_{T_{H}}\left(\mathrm{v}, \tilde{p}_{h}\right) & =0, & & \forall \mathrm{v} \in U_{h}\left(T_{H}\right), \\
-d_{T_{H}}\left(\hat{\psi}_{h}^{ \pm}, q\right) & =-\left( \pm \ell_{T_{H}}^{O^{p}}(q)\right) & \forall q \in M_{h}\left(T_{H}\right),
\end{array}
$$

where

$$
U_{h}^{D}\left(T_{H}\right)=\left\{\left.\mathrm{v}\right|_{T_{H}} \in L_{h}\left(T_{H}\right)|\mathrm{v}|_{\gamma_{T_{H}}}=\left.\hat{\psi}_{H}^{ \pm}\right|_{\gamma_{T_{H}}}, \forall \gamma \in \mathcal{E}\left(T_{H}\right)\right\}
$$

In effect, (69) is simply the affine manifold which imposes on $\hat{\psi}_{h}^{ \pm}$the $H$-mesh adjoint values $\hat{\psi}_{H}^{ \pm}$on the boundary of $T_{H}$; it is important to note that, on $\partial T_{H}$ and $\partial T_{h}$, the bubble function vanishes, so that the trace of $\hat{\psi}_{H}^{ \pm}$on $\partial T_{H}$ is in $U_{h}\left(T_{H}\right)$ - the h-mesh subdomain space. In fact, $(67)-(68)$ indicates that $\hat{\psi}_{h}^{ \pm}$is an incompressible $H^{1}$ semi-norm projection of $\hat{\psi}_{H}^{ \pm}$onto the fine-mesh; $\dot{p}_{h}$ in $(6 T)$ is a "dummy" variable (Lagrange multiplier) which is not used in the remainder of this work. Note that. if $q=1$ in $(68)$. then since 1 is in $M_{H}\left(T_{H}\right)$,

$$
-d_{T_{H}}\left(\hat{\boldsymbol{\psi}}_{h}^{ \pm}, 1\right)=-\int_{\partial \Omega} \hat{\boldsymbol{\psi}}_{H}^{ \pm} \cdot \hat{\mathrm{n}}^{\boldsymbol{\top} T_{H}} \mathrm{~d} s=-d_{T_{H}}\left(\hat{\boldsymbol{\psi}}_{H}^{ \pm} \cdot 1\right)=-\left( \pm \ell_{T_{H}}^{O p}(1)\right)
$$

from (51); recall that $\hat{\mathrm{n}}^{\gamma_{H}}$ is the outward normal on ${ }_{i} T_{H}$ with respect to $T_{H}$. The system (67)-(68) is thus solvable; note the issue of solvability does not arise in (67) because we do not have any Neumann problems - all boundaries are Dirichlet.

For the local subdomain problem, we now look for $\hat{\mathbf{u}}_{T_{H}}^{ \pm} \in \mathcal{L}_{h}\left(T_{H}\right)$. for all $T_{H}$ in $\mathcal{T}_{H}$. such that

$$
\begin{aligned}
& 2 a_{T_{H}}\left(\mathbf{w}, \hat{\mathbf{u}}_{T_{H}}^{ \pm}\right)-d_{T_{H}}\left(\mathbf{w}, \overline{\bar{\pi}}_{T_{H}}^{ \pm}\right)=-\left( \pm \ell_{T_{H}}^{O_{H}}(\mathbf{w})-\ell_{T_{H}}^{N}(\mathbf{w})+a_{T_{H}}\left(\hat{\boldsymbol{\psi}}_{h}^{ \pm}, \mathbf{w}\right)\right. \\
& \left.-d_{T_{H}}\left(\mathbf{w}, \Lambda_{h}^{ \pm}\right)-\left.\sum_{\gamma_{T_{H}} \in \mathcal{E}\left(T_{H}\right)} \sigma_{T_{H}}^{\gamma_{H}} \int_{\gamma_{T_{H}}} \mathbf{w} \cdot \mathbf{y}^{ \pm}\right|_{E\left(\gamma_{T_{H}}\right)} \mathrm{d} s\right), \quad \forall \mathbf{w} \in U_{h}\left(T_{H}\right), \\
& -d_{T_{H}}\left(\hat{\mathbf{u}}_{T_{H}}^{ \pm}, q\right)=0, \quad \forall q \in M_{h}\left(T_{H}\right)
\end{aligned}
$$

To verify solvability, we take $\mathbf{v}=1$ in (59). The right-hand side of (71) then vanishes because $1 \in U_{H}\left(T_{H}\right) \in U_{h}\left(T_{H}\right)$. Note that the construction of $\hat{\psi}_{h}^{ \pm}$is also essential: the equilibrium equation (58) includes $\hat{\psi}_{H}^{ \pm}$, but in (71), $\hat{\psi}_{h}^{ \pm}$appears; however, since $a\left(\hat{\boldsymbol{\psi}}_{h}^{ \pm}, \mathbf{1}\right)=a\left(\hat{\boldsymbol{\psi}}_{H}^{ \pm}, \mathbf{1}\right)=0$, we are still able to ensure solvability.

In a more compact notation, we can introduce two functions $\hat{\mathcal{U}}_{h}^{ \pm} \in W_{h}$ and $\bar{\Pi}_{h}^{ \pm} \in M_{h}$ such that $\left.\hat{\mathcal{U}}_{h}^{ \pm}\right|_{T_{H}}=\hat{\mathrm{u}}_{T_{H}}^{ \pm}$and $\left.\bar{\Pi}_{h}^{ \pm}\right|_{T_{H}}=\bar{\pi}_{T_{H}}^{ \pm}, \forall T_{H} \in \mathcal{T}_{H}$, where $\hat{\mathcal{U}}_{h}^{ \pm}$satisfies

$$
\begin{aligned}
2 a\left(\mathbf{w}, \hat{\mathcal{U}}_{h}^{ \pm}\right)-d\left(\mathbf{w}, \bar{\Pi}_{h}^{ \pm}\right) & =-F^{ \pm}\left(\mathbf{w} ; \hat{\boldsymbol{\psi}}_{h}^{ \pm}, \Lambda_{H}^{ \pm}\right)+b\left(\mathbf{w}, \mathbf{y}^{ \pm}\right), \quad \forall \mathbf{w} \in V_{h}, \\
-d\left(\hat{\mathcal{U}}_{h}^{ \pm}, q\right) & =0, \quad \forall q \in M_{h} .
\end{aligned}
$$


We make several remarks. First, we note that the $K$ systems for $\hat{u}_{T_{H}}^{ \pm}$are completely decoupled, leading to very efficient inversion compared to the original $h$-mesh problem of $(6)-(7)$. This cost reduction is considerable, especially when decoupling the Stokes problem, which is a larger system with a larger bandwidth than an elliptic problem. An additional advantage is that each of these subdomain problems may be easily solved in parallel. Second, an additional constraint is introduced on the subdomain problems to impose the local incompressibility constraint on $\hat{\mathcal{U}}_{h}^{ \pm}$. As we will see, this is not required by the bound theory, however we expect that it improves the accuracy of the bounds. By imposing the incompressibility constraint, which is more expensive, we look for the solutions to local Stokes problems instead of local Poisson problems. We have not yet investigated the latter. Third, note that we solve two (one for each bound) local Stokes problem to project the adjoint onto the $h$-mesh. The cost of this additional solve is small, especially if we use direct solvers, in which case only one $\mathrm{LU}$ decomposition is necessary for both the adjoint and the subsequent velocity calculations.

Finally, we can now calculate the bounds as

$$
\left(s_{h}\right)_{L B}(H)=\eta^{+}
$$

and

$$
\left(s_{h}\right)_{U B}(H)=-\eta^{-}
$$

where

$$
\eta^{ \pm}=-a\left(\hat{\mathcal{U}}_{h}^{ \pm}, \hat{\mathcal{U}}_{h}^{ \pm}\right)-\ell^{N}\left(\hat{\boldsymbol{\psi}}_{h}^{ \pm}\right)
$$

Note that the upper bound can be interpreted as the lower bound in which the output is multiplied by -1 .

\section{Proof of Bounding Properties}

The proof of the bounding properties of $\eta^{ \pm}$is based on classical quadratic duality theory $[28,19]$. The key feature of our approach is the construction of a Lagrangian with a quadratic objective function and linear constraints such that, at stationarity, this Lagrangian evaluates to the output of interest. We first derive an "energy" equality that provides the stabilization in our Lagrangian. We take the test function in (4S)-(49) to be the solution $\left(\mathbf{u}_{h}^{ \pm}, p_{h}\right)$ which yields

$$
\begin{aligned}
a\left(\mathbf{u}_{h}, \mathbf{u}_{h}\right)-d\left(\mathbf{u}_{h}, p_{h}\right) & =\ell^{N}\left(\mathbf{u}_{h}\right), \\
d\left(\mathbf{u}_{h}, q\right) & =0, \quad \forall q \in Y_{h} .
\end{aligned}
$$

Note that (78)-(79) reduces to a quadratic form in $\mathbf{u}_{h}-a\left(\mathbf{u}_{h}, \mathbf{u}_{h}\right)-\ell^{N}\left(\mathbf{u}_{h}\right)=0-$ because the term $d\left(\mathbf{u}_{h}, p_{h}\right)$ is zero. For inhomogeneous Dirichlet boundary conditions, a boundary function would be introduced, as in [20], to directly obtain boundary conditions for the adjoint; the error formulation of $[17,25]$ can also be pursued. 
By adding the output functional to the quadratic form (78) we obtain a function that reduces to $s_{h}=\ell^{O^{v}}\left(\mathbf{u}_{h}\right)+\ell^{O^{p}}\left(p_{h}\right)$ when $\left(\mathbf{v}=\mathbf{u}_{h}, q=p_{h}\right)$. More precisely, we have

$$
\pm s_{h}=\min _{(\mathbf{v}, q) \in \mathcal{S}}\left( \pm \ell^{O^{v}}(\mathbf{v}) \pm \ell^{O^{p}}(q)+a(\mathbf{v}, \mathbf{v})-\ell^{N}(\mathbf{v})\right)
$$

where

$$
\mathcal{S}=\left\{\begin{array}{l|l}
(\mathbf{v}, q) \in W_{h} \times Y_{h} & \begin{array}{l}
a(\boldsymbol{\mu}, \mathbf{v})-d(\boldsymbol{\mu}, q)=\ell^{N}(\boldsymbol{\mu}), \forall \boldsymbol{\mu} \in X_{h}, \\
d(\mathbf{v}, \lambda)=0, \forall \lambda \in Y_{h}, \\
b(\mathbf{v}, \mathrm{t})=0, \forall \mathrm{t} \in \mathcal{Q}^{2}
\end{array}
\end{array}\right\} .
$$

The set of functions $\mathcal{S}$ is a singleton $\left(\mathbf{v}=\mathbf{u}_{h}, q=p_{h}\right)$ equivalent to the solution of the Stokes equations (48)-(49). Note we could replace $W_{h}$ with $V_{h}$, which would yield decoupled Poisson rather than Stokes subproblems, as described in the previous section; we consider here the arguably more accurate choice $W_{h}$.

From a mathematical point of view, the solution to (80) is equivalent to finding the saddlepoint of a Lagrangian, $\mathcal{L}^{ \pm}:(\mathbf{v}, q, \boldsymbol{\mu}, \lambda, \mathbf{t}) \in W_{h} \times Y_{h} \times X_{h} \times Y_{h} \times \mathcal{Q}^{2}$

$$
\begin{aligned}
\mathcal{L}^{ \pm}(\mathbf{v}, q, \boldsymbol{\mu}, \lambda, \mathbf{t}) & = \pm \ell^{O^{\prime \prime}}(\mathbf{v}) \pm \ell^{O^{p}}(q) \\
+a(\mathbf{v}, \mathbf{v})-\ell^{N}(\mathbf{v}) & +a(\boldsymbol{\mu}, \mathbf{v})-d(\boldsymbol{\mu}, q)-\ell^{N}(\boldsymbol{\mu}) \\
& -d(\mathbf{v}, \lambda)-b(\mathbf{v}, \mathbf{t}) .
\end{aligned}
$$

Inserting $F^{ \pm}(\mathbf{v} ; \boldsymbol{\mu}, \lambda)$ from (53) and regrouping terms so that subsequent simplifications are more obvious, we can rewrite ( $\$ 2)$ as

$$
\begin{aligned}
\mathcal{L}^{ \pm}(\mathbf{v}, q, \boldsymbol{\mu}, \lambda, \mathbf{t}) & =\left[-a(\mathbf{v}, \mathbf{v})-\ell^{N}(\boldsymbol{\mu})\right] \\
& +\left[2 a(\mathbf{v}, \mathbf{v})+F^{ \pm}(\mathbf{v} ; \boldsymbol{\mu}, \lambda)-b(\mathbf{v}, \mathbf{t})\right] \\
& +\left[-d(\boldsymbol{\mu}, q) \pm \ell^{O^{p}}(q)\right] .
\end{aligned}
$$

Our first goal is to show that this Lagrangian evaluates to $\eta^{ \pm}$of $(i 7)$ for $(\mathbf{v}, q, \boldsymbol{\mu}, \lambda, \mathbf{t})=$ $\left(\hat{\mathcal{U}}_{h}^{ \pm}, \cdot, \hat{\psi}_{h}^{ \pm}, \Lambda_{h}^{ \pm}, \mathrm{y}^{ \pm}\right)$, where "." represents any value in $Y_{h}$. Proceeding, we obtain

$$
\begin{aligned}
\mathcal{L}^{ \pm}\left(\hat{\mathcal{U}}_{h}^{ \pm}, \cdot, \hat{\boldsymbol{\psi}}_{h}^{ \pm}, \Lambda_{h}^{ \pm}, \mathbf{y}^{ \pm}\right) & =\left[-a\left(\hat{\mathcal{U}}_{h}^{ \pm}, \hat{\mathcal{U}}_{h}^{ \pm}\right)-\ell^{N}\left(\hat{\boldsymbol{\psi}}_{h}^{ \pm}\right)\right] \\
& +\left[2 a\left(\hat{\mathcal{U}}_{h}^{ \pm}, \hat{\mathcal{U}}_{h}^{ \pm}\right)+F^{ \pm}\left(\hat{\mathcal{U}}_{h}^{ \pm} ; \hat{\boldsymbol{\psi}}_{h}^{ \pm}, \Lambda_{h}^{ \pm}\right)-b\left(\hat{\mathcal{U}}_{h}^{ \pm}, \mathbf{y}^{ \pm}\right)\right] \\
& +\left[-d\left(\hat{\boldsymbol{\psi}}_{h}^{ \pm}, \cdot\right) \pm \ell^{O^{p}}(\cdot)\right] .
\end{aligned}
$$

We immediately see that the first bracket of (84) equals $\eta^{ \pm}$, where we recall that

$$
\eta^{ \pm}=-a\left(\hat{\mathcal{U}}_{h}^{ \pm}, \hat{\mathcal{U}}_{h}^{ \pm}\right)-\ell^{N}\left(\hat{\psi}_{h}^{ \pm}\right)
$$

It remains to show that all the other terms in (84) vanish. We observe that the second bracket in (84) is, from (73), $d\left(\hat{\mathcal{U}}_{h}^{ \pm}, \bar{\Pi}_{h}^{ \pm}\right)$which is zero thanks to ( 74$)$. Finally, the last bracket in (84) 
vanishes due to the construction of the adjoint, since we have imposed $-d\left(\hat{\psi}_{h}^{ \pm}, \cdot\right) \pm \ell^{O^{p}}(\cdot)=0$ in (68).

We conclude that

$$
\eta^{ \pm}=\mathcal{L}^{ \pm}\left(\hat{\mathcal{U}}_{h}^{ \pm}, \cdot, \hat{\psi}_{h}^{ \pm}, \Lambda_{h}^{ \pm}, \mathrm{y}^{ \pm}\right)
$$

It then follows from the classical quadratic linear duality theory that

$$
\eta^{ \pm} \leq \pm s_{h}, \text { or } \eta^{+} \leq s_{h} \leq-\eta^{-},
$$

if

$$
\mathcal{L}^{ \pm}\left(\hat{\mathcal{U}}_{h}^{ \pm}, \cdot, \hat{\boldsymbol{\psi}}_{h}^{ \pm}, \Lambda_{h}^{ \pm}, \mathrm{y}^{ \pm}\right)=\min _{\mathrm{v} \in \mathfrak{W}_{h}} \mathcal{L}^{ \pm}\left(\mathrm{v}, \cdot, \hat{\psi}_{h}^{ \pm}, \Lambda_{h}^{ \pm}, \mathrm{y}^{ \pm}\right)
$$

To demonstrate (88), we expand our Lagrangian (82) for $\mathrm{v}=\hat{\mathcal{U}}_{h}^{ \pm}+\mathbf{w}, \boldsymbol{\mu}=\hat{\boldsymbol{\psi}}_{h}^{ \pm}, \lambda=$ $\Lambda_{h}^{ \pm}, \mathrm{t}=\mathrm{y}^{ \pm}$, to obtain,

$$
\begin{aligned}
\mathcal{L}^{ \pm}\left(\hat{\mathcal{U}}_{h}^{ \pm}+\mathbf{w}, \cdot \hat{\psi}_{h}^{ \pm}, \Lambda_{h}^{ \pm}, \mathbf{y}^{ \pm}\right) & =\mathcal{L}^{ \pm}\left(\hat{\mathcal{U}}_{h}^{ \pm}, \cdot, \hat{\psi}_{h}^{ \pm}, \Lambda_{h}^{ \pm}, \mathbf{y}^{ \pm}\right) \\
+\left[2 a\left(\mathbf{w}, \hat{\mathcal{U}}_{h}^{ \pm}\right)+F^{ \pm}\left(\mathbf{w} ; \hat{\psi}_{h}^{ \pm}, \Lambda_{h}^{ \pm}\right)-b\left(\mathbf{w}, \mathbf{y}^{ \pm}\right)\right] & +\left[-d\left(\hat{\psi}_{h}^{ \pm}, \cdot\right) \pm \ell^{O^{p}}(\cdot)\right] \\
& +a(\mathbf{w}, \mathbf{w}), \quad \forall \mathbf{w} \in W_{h} .
\end{aligned}
$$

We observe that all the terms linear in $\mathbf{w}$ (the first bracket) reduce to $d\left(\mathbf{w}, \bar{\Pi}_{h}^{ \pm}\right)$from ( 73 ), which vanishes since $\mathbf{w} \in W_{h} \subset V_{h}$ is incompressible. The terms $-d\left(\hat{\psi}_{h}^{ \pm} \cdot \cdot\right) \pm \ell^{O^{p}}(\cdot)$ (the second bracket) also vanish thanks to the construction of the adjoint (67)-(68). The remaining term $a(\mathbf{w}, \mathbf{w})$ is positive semi-definite, which thus proves (88). Note that it is the energy equality which allows us to consider non-exact Lagrange multipliers and still provide non-infinite bounds.

More precisely, to avoid meaningless bounds we need to rerify that when minimizing our augmented Lagrangian we do not obtain $-\infty$. To this end. two main concerns must be addressed. First, solvability of (73)-(74) is essential. Without solvability the terms on the right-hand side could tend to infinity as the test function tends to infinity. Second. equilibration between $-d\left(\hat{\boldsymbol{\psi}}_{h}^{ \pm}, q\right)$ and $\pm \ell^{O^{P}}(q)$ is also essential, because these terms are not controlled by any quadratic stabilization. Because both of the above conditions are satisfied we are guaranteed non-infinite bounds. However, there is nothing in the presentation that proves that the bounds should be sharp. For the moment, we can suggest that, since $\Psi_{H}^{ \pm}, \Lambda_{H}^{ \pm}$ and $\mathrm{y}^{ \pm}$are the saddlepoints of the $H$-mesh approximation to our Lagrangian, they should thus be close enough to the $h$-mesh saddlepoint to yield good bounds.

\section{Optimal Stabilization Parameter}

In this section we present a procedure by which to improve the sharpness of the bounds. To this end, we introduce a positive real number, $\kappa$, to scale our output $s$, and we look for the bounds to this scaled output. We then provide a procedure by which to calculate the optimal $\kappa$, that is, the $k$ that will yield the sharpest bounds; to be more precise, we maximize our 
lower bound, and minimize our upper bound. A different but equivalent approach in which we scale the entire energy equality (78)-(79) is presented in $\mathbf{R}^{1}$ in $[19,29]$.

Our strategy to find the optimal $\kappa$ is to write all variables as linear functions in $\kappa$, and then derive the bounds as a function of $k$. This procedure does not change the bounding theory and our bounds remain rigorous; indeed, our choices of Lagrange candidates are still valid even if the adjoint and the hybrid flux are decomposed into different contributions. The key is that these candidates must remain in the appropriate spaces, so some attention must be given to the boundary conditions.

First, we decompose $\hat{\psi}_{H}^{ \pm}$and $\Lambda_{H}^{ \pm}$as

$$
\begin{aligned}
& \hat{\psi}_{H}^{ \pm}=\hat{\psi}_{H}^{0 \pm}+\kappa \hat{\psi}_{H}^{1 \pm}, \\
& \Lambda_{H}^{ \pm}=\Lambda_{H}^{0 \pm}+\kappa \Lambda_{H}^{1 \pm},
\end{aligned}
$$

where $\hat{\psi}_{H}^{0 \pm} \in X_{H}$ satisfies

$$
\begin{aligned}
a\left(\hat{\boldsymbol{\psi}}_{H}^{0 \pm}, \mathbf{w}\right)-d\left(\mathbf{w}, \Lambda_{H}^{0 \pm}\right) & =-\left(2 a\left(\mathbf{w}, \mathbf{u}_{H}\right)-l^{Y}(\mathbf{w})\right) . \quad \forall \mathbf{w} \in X_{H} \\
-d\left(\hat{\boldsymbol{\psi}}_{H}^{0 \pm}, q\right) & =0, \quad \forall q \in Y_{H},
\end{aligned}
$$

and $\hat{\psi}_{H}^{1 \pm} \in X_{H}$ satisfies

$$
\begin{aligned}
a\left(\hat{\boldsymbol{\psi}}_{H}^{1 \pm}, \mathbf{w}\right)-d\left(\mathbf{w}, \Lambda_{H}^{1 \pm}\right) & =-\left( \pm \ell^{O^{v}}(\mathbf{w})\right) . \quad \forall \mathbf{w} \in X_{H}, \\
-d\left(\hat{\boldsymbol{\psi}}_{H}^{1 \pm}, q\right) & =-\left( \pm \ell^{O^{P}}(q)\right) . \quad \forall q \in Y_{H} .
\end{aligned}
$$

Note that $\mathbf{u}_{H}$, the solution to (48), only appears on the right-hand side of the equation. In fact, in both equations the operator is identical, and we can take advantage of this fact for direct solvers. We now write $\hat{\psi}_{h}^{ \pm}$as

$$
\hat{\boldsymbol{\psi}}_{h}^{ \pm}=\hat{\boldsymbol{\psi}}_{h}^{0 \pm}+\kappa \hat{\boldsymbol{\psi}}_{h}^{1 \pm},
$$

which needs to satisfy, for each element $T_{H}$ on the $H$-mesh.

$$
\begin{array}{rlrl}
a_{T_{H}}\left(\mathbf{w}, \hat{\boldsymbol{\psi}}_{h}^{0 \pm}-\hat{\boldsymbol{\psi}}_{H}^{0 \pm}\right)-d_{T_{H}}\left(\mathbf{w}, \tilde{p}_{h}^{0}\right) & =0, & & \forall \mathbf{w} \in X_{H}, \\
-d_{T_{H}}\left(\hat{\boldsymbol{\psi}}_{h}^{0 \pm}, q\right) & =0, & \forall q \in Y_{H},
\end{array}
$$

and

$$
\begin{aligned}
a_{T_{H}}\left(\mathbf{w}, \hat{\boldsymbol{\psi}}_{h}^{1 \pm}-\hat{\psi}_{H}^{1 \pm}\right)-d_{T_{H}}\left(\mathbf{w}, \tilde{p}_{h}^{1}\right) & =0 \quad \forall \mathbf{w} \in X_{H}, \\
-d_{T_{H}}\left(\hat{\psi}_{h}^{1 \pm}, q\right) & =-\left( \pm \ell_{T_{H}}^{Q^{p}}(q)\right), \quad \forall q \in Y_{H} .
\end{aligned}
$$

Based on similar arguments as in Section 5, the boundary conditions for $\hat{\psi}_{h}^{0 \pm}-\hat{\psi}_{H}^{0 \pm}$ and $\hat{\boldsymbol{\psi}}_{h}^{1 \pm}-\hat{\boldsymbol{\psi}}_{H}^{1 \pm}$ are homogeneous Dirichlet. These two set of equations are similar to $(67)-(68)$ in all respects; we force continuity of the adjoint across the subdomain boundaries, and we impose an incompressibility constraint in the interior of each subdomain. 
We now present the $\kappa$ decomposition of the hybrid flux. First, we need to define two functions $F^{0 \pm}(v ; \mathcal{F}, \mathcal{P})$ and $F^{1 \pm}(v ; \mathcal{F}, \mathcal{P})$. In particular, for any two functions $\mathcal{F}$ and $\mathcal{P}$ in $\mathcal{X}^{*}$ and $\mathcal{Y}$, we define for all $v \in \mathcal{X}^{*}$

$$
\begin{aligned}
& F^{0 \pm}(\mathrm{v} ; \mathcal{F}, \mathcal{P})=\sum_{T_{H} \in \mathcal{T}_{H}} F_{T_{H}}^{0 \pm}\left(\left.\mathbf{v}\right|_{T_{H}} ; \mathcal{F}, \mathcal{P}\right), \\
& F^{1 \pm}(\mathrm{v} ; \mathcal{F}, \mathcal{P})=\sum_{T_{H} \in \mathcal{T}_{H}} F_{T_{H}}^{1 \pm}\left(\left.\mathbf{v}\right|_{T_{H}} ; \mathcal{F}, \mathcal{P}\right),
\end{aligned}
$$

where

$$
\begin{aligned}
& F_{T_{H}}^{0 \pm}(\mathbf{v} ; \mathcal{F}, \mathcal{P})=a_{T_{H}}(\mathcal{F}, \mathbf{v})-d_{T_{H}}(\mathbf{v}, \mathcal{P})-\ell_{T_{H}}^{N}(\mathbf{v}) \\
& F_{T_{H}}^{1 \pm}(\mathbf{v} ; \mathcal{F}, \mathcal{P})=a_{T_{H}}(\mathcal{F}, \mathbf{v})-d_{T_{H}}(\mathbf{v}, \mathcal{P}) \pm \ell_{T_{H}}^{O^{v}}(\mathbf{v})
\end{aligned}
$$

Finally, we introduce

$$
\mathrm{y}^{ \pm}=\mathrm{y}^{0 \pm}+\kappa \mathrm{y}^{1 \pm} .
$$

As in Section 4.2, we solve, for all $T_{H}$ in $\mathcal{T}_{H}$, the following equations.

$$
\begin{aligned}
& \left.\sum_{\gamma_{T_{H}} \in \mathcal{E}\left(T_{H}\right)} \sigma_{T_{H}}^{\gamma_{T_{H}}} \int_{\gamma_{T_{H}}} \mathrm{v} \cdot \mathrm{y}^{0 \pm}\right|_{E\left(r_{T_{H}}\right)} \mathrm{d} s=2 a_{T_{H}}\left(\mathrm{v}, u_{H}^{ \pm}\right)-F_{T_{H}}^{0 \pm}\left(\mathrm{v}: \hat{\psi}_{H}^{0 \pm} \cdot .1_{H}^{0 \pm}\right), \\
& \forall \mathrm{v} \in U_{H}\left(T_{H}\right) . \\
& \left.\sum_{\gamma_{T_{H}} \in \mathcal{E}\left(T_{H}\right)} \sigma_{T_{H}}^{\gamma_{T_{H}}} \int_{\gamma_{T_{H}}} \mathbf{v} \cdot \mathbf{y}^{1 \pm}\right|_{E\left(\gamma_{T_{H}}\right)} \mathrm{d} s=-F_{T_{H}}^{1 \pm}\left(\mathbf{v} ; \hat{\boldsymbol{\psi}}_{H}^{1 \pm}, \Lambda_{H}^{1 \pm}\right), \quad \forall \mathbf{v} \in U_{H}\left(T_{H}\right)
\end{aligned}
$$

We can now solve the $h$-mesh problems,

$$
\begin{aligned}
2 a\left(\mathbf{w}, \hat{\mathbf{u}}_{h}^{0 \pm}\right)-d\left(\mathbf{w}, \hat{\Pi}_{h}^{0 \pm}\right) & =-F^{0 \pm}\left(\mathbf{w} ; \hat{\psi}_{h}^{0 \pm} \cdot \Lambda_{h}^{0 \pm}\right)+b\left(\mathbf{w} \cdot \mathbf{y}^{0 \pm}\right), \\
-d\left(\hat{\mathbf{u}}_{h}^{0 \pm}, q\right) & =0, \quad \forall(\mathbf{w}, q) \in X_{H} \times Y_{H} .
\end{aligned}
$$

and

$$
\begin{aligned}
2 a\left(\mathbf{w}, \hat{\mathbf{u}}_{h}^{1 \pm}\right)-d\left(\mathbf{w}, \hat{\Pi}_{h}^{1 \pm}\right) & =-F^{1 \pm}\left(\mathbf{w} ; \hat{\boldsymbol{\psi}}_{h}^{1 \pm}, \Lambda_{h}^{1 \pm}\right)+b\left(\mathbf{w}, \mathbf{y}^{1 \pm}\right), \\
-d\left(\hat{\mathbf{u}}_{h}^{1 \pm}, q\right) & =0, \quad \forall(\mathbf{w}, q) \in X_{H} \times Y_{H}
\end{aligned}
$$

We will not address solvability of (108)-(109) and (110)-(111) as it follows our usual proof (see Section 4.3).

Using the same derivation as in ( $7 i)$, the bounds can be expressed as

$$
\begin{aligned}
\eta^{ \pm}(\kappa)= & -\frac{1}{\kappa}\left(a\left(\hat{\mathbf{u}}_{h}^{0 \pm}+\kappa \hat{\mathbf{u}}_{h}^{1 \pm}, \hat{\mathbf{u}}_{h}^{0 \pm}+\kappa \hat{\mathbf{u}}_{h}^{1 \pm}\right)+\ell^{N}\left(\hat{\boldsymbol{\psi}}_{h}^{0 \pm}+\kappa \hat{\psi}_{h}^{1 \pm}\right)\right) \\
= & -\frac{1}{\kappa}\left(a\left(\hat{\mathbf{u}}_{h}^{0 \pm}, \hat{\mathbf{u}}_{h}^{0 \pm}\right)+\ell^{N}\left(\hat{\boldsymbol{\psi}}_{h}^{0 \pm}\right)\right) \\
& -2 a\left(\hat{\mathbf{u}}_{h}^{0 \pm}, \hat{\mathbf{u}}_{h}^{1 \pm}\right)-\ell^{N}\left(\hat{\psi}_{h}^{1 \pm}\right)-\kappa a\left(\hat{\mathbf{u}}_{h}^{1 \pm}, \hat{\mathbf{u}}_{h}^{1 \pm}\right) .
\end{aligned}
$$


Differentiating with respect to $\kappa$, we find

$$
\begin{aligned}
& \eta_{\kappa}^{ \pm}(\kappa)=\frac{1}{\kappa^{2}}\left(a\left(\hat{\mathbf{u}}_{h}^{0 \pm}, \hat{\mathbf{u}}_{h}^{0 \pm}\right)+\ell^{N}\left(\hat{\psi}_{h}^{0 \pm}\right)\right)-a\left(\hat{\mathbf{u}}_{h}^{1 \pm}, \hat{\mathbf{u}}_{h}^{1 \pm}\right), \\
& \eta_{\kappa \kappa}^{ \pm}(\kappa)=-\frac{2}{\kappa^{3}}\left(a\left(\hat{\mathbf{u}}_{h}^{0 \pm}, \hat{\mathbf{u}}_{h}^{0 \pm}\right)+\ell^{N}\left(\hat{\psi}_{h}^{0 \pm}\right)\right) .
\end{aligned}
$$

To optimize our bounds we require $\eta_{\kappa}^{ \pm}\left(\kappa^{* \pm}\right)=0$, which yields,

$$
\kappa^{* \pm}=\sqrt{\frac{a\left(\hat{\mathbf{u}}_{h}^{0 \pm}, \hat{\mathbf{u}}_{h}^{0 \pm}\right)+\ell^{N}\left(\hat{\boldsymbol{\psi}}_{h}^{0 \pm}\right)}{a\left(\hat{\mathbf{u}}_{h}^{1 \pm}, \hat{\mathbf{u}}_{h}^{1 \pm}\right)}} .
$$

To prove that $\kappa$ is a maximum, we proceed as follows: we first recall that $\eta^{ \pm}$is a lower bound to $\pm s_{h}$. It follows that the terms in $\frac{1}{\kappa}$ must be positive so that our lower bound does not go to $+\infty$ as $\kappa$ decreases. These same terms also enter in the second derivative (and the radical) making the second derivative negative for all positive values of $\kappa$ (and the argument of the radical positive). The arguments are somewhat more transparent with the error formulation of $[17]$.

We will now make some remarks concerning computational cost; we wish to show that we need only two subdomain solves rather than four to calculate the bounds for the optimal stabilization parameter $\kappa^{*}$. It is obvious that the numerator is the same in both the upper and the lower bound calculations because it does not depend on the output functional. In addition, we can show from (94)-(95) and (99)-(100) that $\hat{\psi}_{h}^{1+}=-\hat{\psi}_{h}^{1-}$. Furthermore, we note that the right-hand side of (110) only differs by a sign when replacing $\hat{\psi}_{h}^{1+}$ by $-\hat{\psi}_{h}^{1-}$, which leads to $\hat{\mathbf{u}}_{h}^{1+}=-\hat{\mathrm{u}}_{h}^{1-}$. Finally, because $a($,$) is a symmetric positive-semidefinite form.$ $a\left(\hat{\mathbf{u}}_{h}^{1+}, \hat{\mathbf{u}}_{h}^{1+}\right)=a\left(\hat{\mathbf{u}}_{h}^{1-}, \hat{\mathbf{u}}_{h}^{1-}\right)$, and thus the denominator of $(116)$ is the same for both the upper and the lower bounds. From the above arguments, we obtain that $\kappa^{*+}=\kappa^{*-} \equiv \kappa^{*}$.

It follows that, in fact, we only need to perform two subdomain solves to compute our optimized bounds, just as in the non-optimized case. For clarity we summarize the relevant identities: $\hat{\mathbf{u}}_{h}^{0+}=\hat{\mathbf{u}}_{h}^{0-}, \hat{\mathbf{u}}_{h}^{1+}=-\hat{\mathbf{u}}_{h}^{1-}, \hat{\boldsymbol{\psi}}_{h}^{0+}=\hat{\psi}_{h}^{0-}$, and $\hat{\psi}_{h}^{1+}=-\hat{\boldsymbol{\psi}}_{h}^{1-}$. These identities also lead to an interesting property that the average of the bounds is not affected by $\kappa$. The average of the bounds is given by

$$
\begin{aligned}
\frac{1}{2}\left(\eta^{+}-\eta^{-}\right)= & -\frac{1}{2 \kappa}\left(a\left(\hat{\mathbf{u}}_{h}^{0+}, \hat{\mathbf{u}}_{h}^{0+}\right)+\ell^{N}\left(\hat{\boldsymbol{\psi}}_{h}^{0+}\right)\right. \\
& \left.-a\left(\hat{\mathbf{u}}_{h}^{0-}, \hat{\mathbf{u}}_{h}^{0-}\right)-\ell^{N}\left(\hat{\boldsymbol{\psi}}_{h}^{0-}\right)\right) \\
& -a\left(\hat{\mathbf{u}}_{h}^{0+}, \hat{\mathbf{u}}_{h}^{1+}\right)+a\left(\hat{\mathbf{u}}_{h}^{0-}, \hat{\mathbf{u}}_{h}^{1-}\right) \\
& -\frac{1}{2} \ell^{N}\left(\hat{\boldsymbol{\psi}}_{h}^{1+}\right)+\frac{1}{2} \ell^{N}\left(\hat{\boldsymbol{\psi}}_{h}^{1-}\right) \\
& -\frac{\kappa}{2}\left(a\left(\hat{\mathbf{u}}_{h}^{1+}, \hat{\mathbf{u}}_{h}^{1+}\right)-a\left(\hat{\mathbf{u}}_{h}^{1-}, \hat{\mathbf{u}}_{h}^{1-}\right)\right) .
\end{aligned}
$$

From the above identities we observe that the terms in $\frac{1}{2 \kappa}$ and $\frac{\kappa}{2}$ all vanish. After replacing the remaining $\hat{\mathbf{u}}_{h}^{1-}$ and $\hat{\psi}_{h}^{1-}$ by $-\hat{\mathbf{u}}_{h}^{1+}$ and $-\hat{\boldsymbol{\psi}}_{h}^{1+}$, respectively, we obtain

$$
\frac{1}{2}\left(\eta^{+}-\eta^{-}\right)=-2 a\left(\hat{\mathbf{u}}_{h}^{1+}, \hat{\mathbf{u}}_{h}^{1+}\right)-\ell^{N}\left(\hat{\boldsymbol{\psi}}_{h}^{1+}\right)
$$




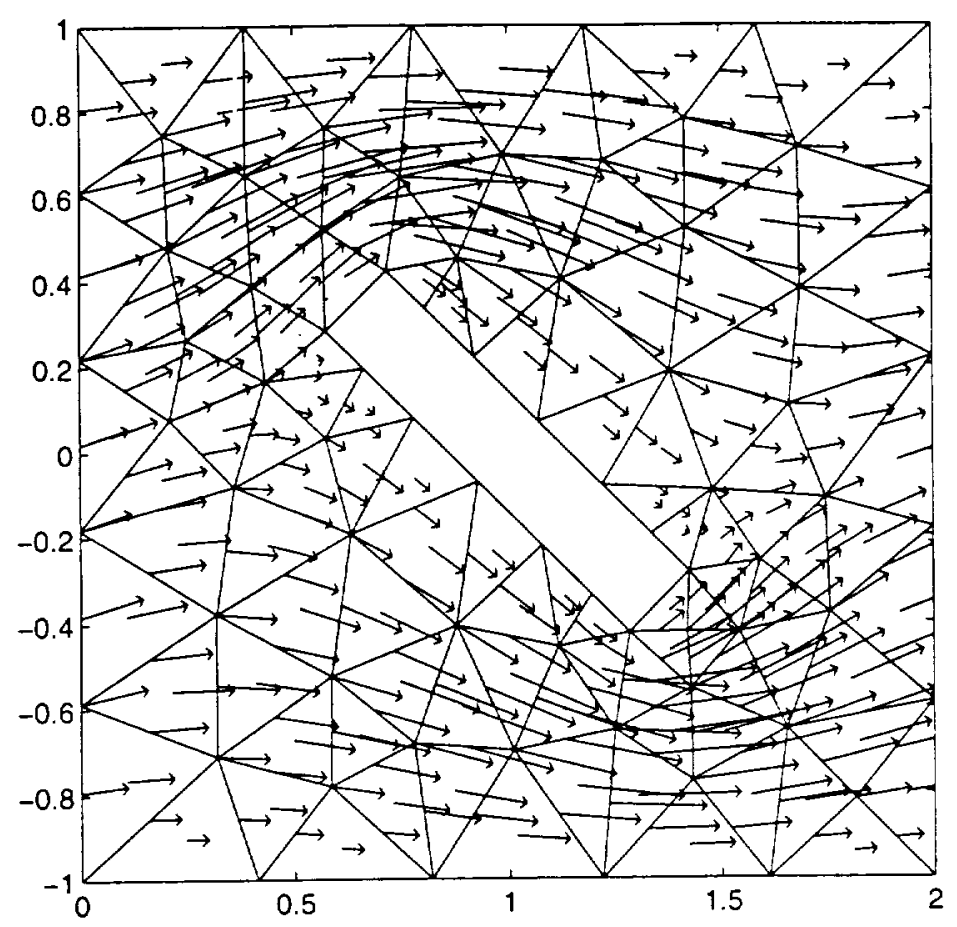

Figure 2: Velocity field solution for $\mathcal{T}_{H}=\mathcal{T}_{\left(H_{o}, 1\right)}$.

thus proving the desired result.

\section{$7 \quad$ Numerical Results}

We present here results for the Stokes problem for a periodic domain (Figure 1) in which the flow is driven by a pressure gradient. The velocity field solution of this problem is shown in Figure 2 for the coarsest mesh, $\mathcal{T}_{\left(H_{o}, 1\right)}$. The triangulations investigated, $\mathcal{T}_{\left(H_{o}, R\right)}$, are uniform refinements of the coarsest mesh $\mathcal{T}_{\left(H_{o}, 1\right)}$ shown in Figure $3 \mathrm{a}$. The $H$-meshes, $\mathcal{T}_{H}$, correspond to $\mathcal{T}_{\left(H_{o}, R\right)}, R=1,2,3,4,6$, and the truth $h$-mesh corresponds to $\mathcal{T}_{h}=\mathcal{T}_{\left(H_{o}, 12\right)} ; \mathcal{T}_{h}$ is shown in Figure $3 \mathrm{~b}$. Note that, for all the refinement values of $R$ considered, we satisfy $X_{H} \subset X_{h}$, as required by the theory. We shall denote the effective working-approximation element size associated with triangulation $\mathcal{T}_{H}=\mathcal{T}_{\left(H_{0}, R\right)}$ by $H \equiv 1 / R$.

Two outputs are investigated, as defined in $(10)$ : the flowrate, $s^{(1)}$, and the lift force on the body, $s^{(2)}$. The test function $\chi\left(\in \mathcal{H}^{1}(\Omega) \otimes \mathcal{H}^{1}(\Omega)\right)$ used in the lift functional (13) is defined to be continuous and piecewise linear over the $T_{H}$ in $\mathcal{T}_{\left(H_{0}, 1\right)}$ with

$$
\begin{aligned}
\chi=0, & \text { in } \Omega \backslash \Omega^{\prime}, \\
\chi=0, & \text { on } \Gamma_{i}, i=\{1,2,3,4\}, \\
\chi \cdot \mathbf{e}_{2}=1, & \text { on } \Gamma_{5},
\end{aligned}
$$


where $\Omega^{\prime}$ contains all the elements of $\mathcal{T}_{\left(H_{0}, 1\right)}$ that have an edge on $\Gamma_{5}$. Another choice of (incompressible) $\chi$ is presented in [21], which yields almost identical results but at a higher computational cost.

The objective here is to rigorously bound the output associated with $\mathcal{T}_{\left(H_{0}, 12\right)}$. To this end, different $H$-meshes can be exploited. It is obvious that the cost of the bound calculations increases as finer $H$-meshes are used, that is. as $R$ increases for $\mathcal{T}_{\left(H_{0}, R\right)}$. However, finer $H$-meshes also lead to sharper bounds because the adjoint and the hybrid flux are more accurately approximated. In fact, an adaptive procedure similar to [24] could be developed to efficiently produce a $H$-mesh and associated bound gap within a desired value.

We can easily relate our hierarchical mesh procedure for calculation of the bounds to engineering design procedures based upon a hierarchy of numerical approximations. In fact, the first discretization, here the $H$-mesh, is a "working" coarse mesh approximation which is relatively inexpensive, but which generates solutions and associated outputs $s_{H}$ that are deemed sufficiently accurate for the purposes of "preliminary" analysis. The second discretization. here the $h$-mesh, is a "truth" mesh which produces a solution and associated outputs $s_{h}$ for which $\left|s_{h}-s\right|$ is assumed negligibly small. The $h$-discretization serves to verify the prediction of the $H$-discretization, either prior to design, as in a validated-surrogates framework [32], during design, as in the trust-region optimization techniques [6], or after design, as final confirmation of the anticipated performance. Our bound procedure provides reliability of the truth mesh but at much lower cost.

We plot in Figure $4 \mathrm{a}$ and $4 \mathrm{~b}\left(s_{h}\right)_{i B}^{*} / s_{h},\left(s_{h}\right)_{p r \varepsilon}^{*} / s_{h},\left(s_{h}\right)_{L B}^{*} / s_{h}$, and $s_{H} / s_{h}$ as a function of (effective) $H$ for, respectively, $s^{(1)}$ (flowrate), and $s^{(2)}$ (lift force). The average of the lower and upper bounds is denoted by $\left(s_{h}\right)_{p r e}^{*}$. For the coarsest mesh. we observe that the upper bounds for both outputs are within $+15 \%$. The accuracy of the lower bounds depends on the output considered. For the flowrate output. $s^{(1)}$, the lower bounds are within $-5 \%$ and almost equal to $s_{H}$; in fact, in this case we have a "weak" compliance. (By compliance we refer to the property that the output calculated on the $H$-mesh is equal to the lower bound, $\left(s_{h}\right)_{L B}=\eta^{+}=s_{H}$, which occurs when (i) the inhomogeneity of the weak form equals the output functional, (ii) the boundary conditions are homogeneous Dirichlet, and (iii) the operator of the problem considered is symmetric [21].) For $s^{(2)}$, the lower bounds are within $-20 \%$ of $s_{h}$ calculated on $\mathcal{T}_{\left(H_{0}, 1\right)}$. We also observe that, for a refinement of two, both upper and lower bounds are well within $\pm 10 \%$. Recall that one of main advantages of the bounds is the certainty that $s_{h}$ does indeed lie within the calculated values. In practice, the "working" mesh should be constructed sufficiently accurate (considerably more so than our $\mathcal{T}_{\left(H_{o}, 1\right)}$ used here).

In Figure 5a and 5b we plot $e_{U B}^{*}=\log \left|\left(s_{h}\right)_{U B}^{*}-s_{h}\right|, e_{L B}^{*}=\log \left|\left(s_{h}\right)_{L B}^{*}-s_{h}\right|, \quad e_{p r e}^{*}=$ $\log \left|\left(s_{h}\right)_{\text {pre }}^{*}-s_{h}\right|$, and $e_{H}=\log \left|s_{H}-s_{h}\right|$ as a function of $\log H$ for $s^{(1)}$ and $s^{(2)}$, respectively. For $s^{(1)},\left(s_{h}\right)_{L B}^{*}$ and $s_{H}$ appear to converge to $s_{h}$ as $O\left(H^{1.5}\right)$ as $H \rightarrow h$. We would expect, for a smooth solution, that $s_{H}$ will converge at least as fast as $O\left(H^{2}\right)$, and no doubt faster. The corner singularities are most probably responsible for $s_{H}$ converging to $s_{h}$ only as $O\left(H^{1.5}\right)$. Note that from our "weak" compliance analysis in [21] the hybrid fluxes are zero, and we therefore rule out any error contribution from that calculation for the lower bound; as expected, we obtain the same convergence rates for both $\left(s_{h}\right)_{L B}^{*}$ and $s_{H}$. Now, considering the 

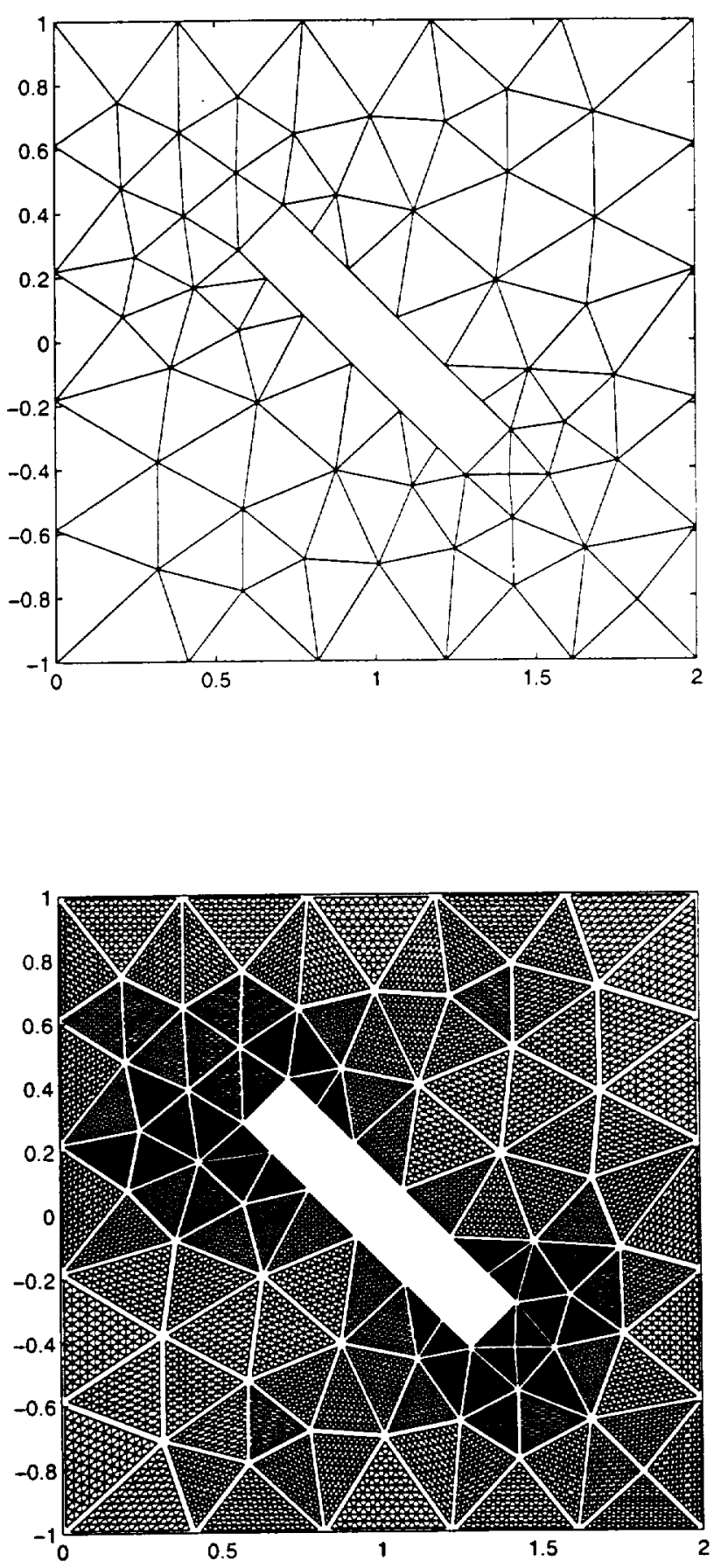

Figure 3: (a) Coarsest working mesh $\mathcal{T}_{H}=\mathcal{T}_{\left(H_{o}, 1\right)}$, and (b) truth mesh $\mathcal{T}_{H}=\mathcal{T}_{\left(H_{o}, 12\right)}$. 

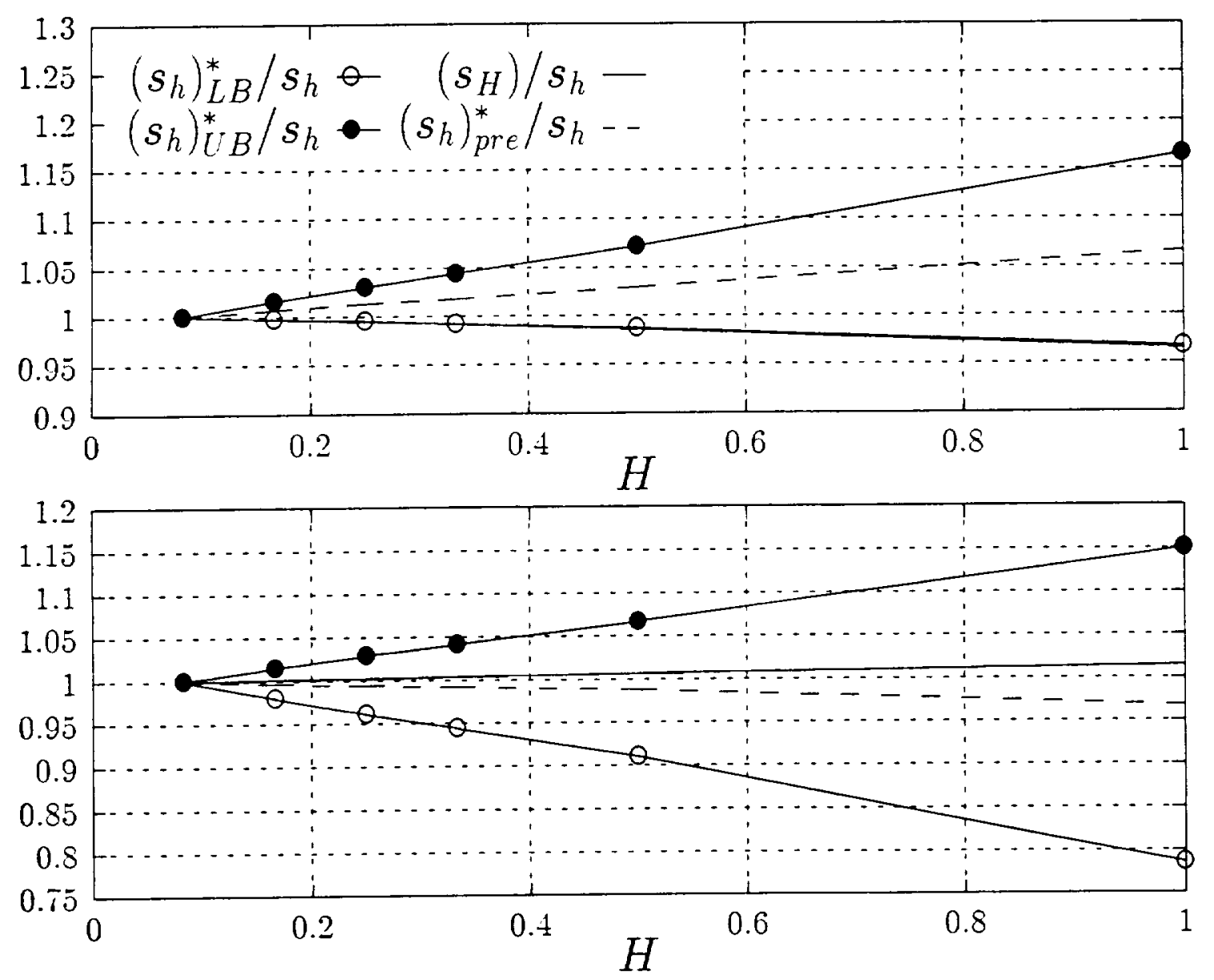

Figure 4: Plots of $\left(s_{h}\right)_{U B}^{*} / s_{h},\left(s_{h}\right)_{p r e}^{*} / s_{h},\left(s_{h}\right)_{L B}^{*} / s_{h}$, and $s_{H} / s_{h}$ as a function of (effective) $\mathrm{H}$ for $(a$, top $) s^{(1)}$, the flow rate and $\left(b\right.$, bottom) $s^{(2)}$, the lift force. 
convergence of $\left(s_{h}\right)_{U B}^{*}$ (still for the flowrate output), we note that we achieve only $O\left(H^{1.3}\right)$ compared to $O\left(H^{1.5}\right)$ for $\left(s_{h}\right)_{L B}^{*}$. We believe that this may be caused by the hybrid flux approximation - unfortunately preliminary work with a $\mathbf{P}_{1}$ initial approximation did not indicate any improvements [21]. Considering now $s^{(2)}$, the quantities $\left(s_{h}\right)_{U B}^{*},\left(s_{h}\right)_{L B}^{*}$ and $\left(s_{h}\right)_{\text {pre }}^{*}$ all converge at the previous lower rate, $O\left(H^{1.3}\right)$, and $s_{H}$ converges at the same rate as for the flowrate, $O\left(H^{1.5}\right)$. The same comments regarding the hybrid flux and the singularity can also be evoked for the lift output, $s^{(2)}$, and no doubt explain the convergence rate results.

The bounds presented here reflect the use of the scaling parameter $\kappa$ described in Section 6 . For the flowrate output, $\kappa=1$ is optimal for all $H$ (again due to the compliance result), while for the lift output $\kappa^{*}$ tends to 0.0886 as $R$ increases. Note that the choice of $\chi$ does not influence significantly the accuracy and convergence of the bounds, as shown in [21].

We conclude with a few suggestions to improve the bounds for outputs of the Stokes problem. First, closer examination of the hybrid flux calculations is warranted; in particular, investigation of a $\mathrm{P}_{1}$ initial approximation of the hybrid flux should improve the convergence rate of the bounds. Second, implementing the bounds technique within the stress formulation of the Stokes equations will allow for cleaner derivation of the lift linear functional. And finally, additional application to more relevant engineering problems will be presented in future papers.

\section{Acknowledgments}

This work was supported by DARPA and ONR under Grant N00014-91-J-1S89, AFOSR under Grant F49620-97-1-0052, NASA Langley Research Center under Grants NAG1-1613, NAG1-1978, and NASA Lewis Research Center under Grant NCC 3-438. 

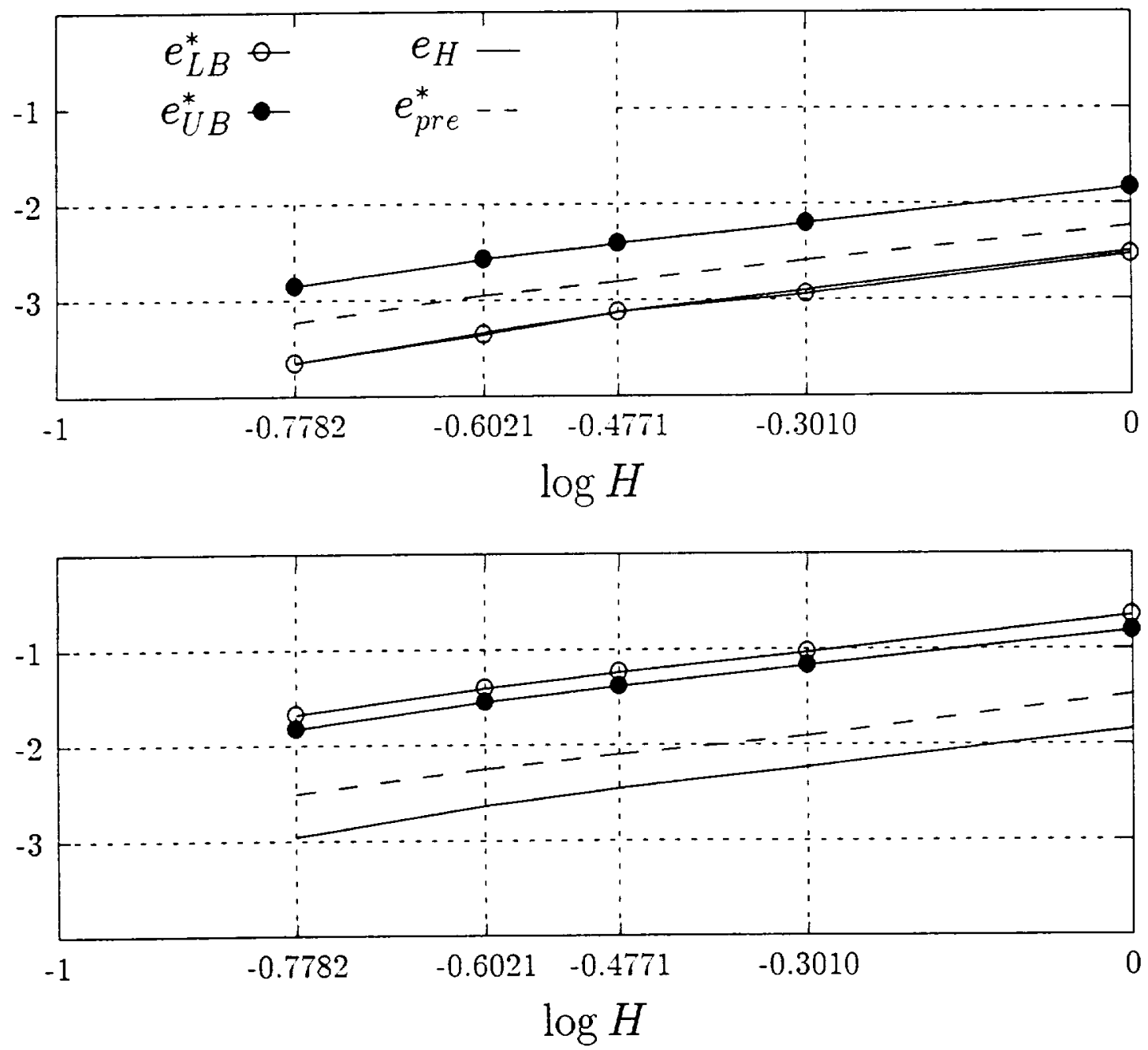

Figure 5: Plots of $e_{U B}^{*}=\log \left|\left(s_{h}\right)_{U B}^{*}-s_{h}\right|, e_{L B}^{*}=\log \left|\left(s_{h}\right)_{L B}^{*}-s_{h}\right|, e_{p r e}^{*}=\log \left|\left(s_{h}\right)_{p r e}^{*}-s_{h}\right|$, and $e_{H}=\log \left|s_{H}-s_{h}\right|$ as a function of $\log H$ for (a, top) $s^{(1)}$ the flowrate and (b, bottom) $s^{(2)}$ the lift force. 


\section{References}

[1] R.A. Adams, Sobolev Spaces, Academic Press, New York, 1975.

[2] D.N. Arnold, F. Brezzi, and M. Fortin. A stable finite element for the Stokes Equations, Calcolo, 21(1984), pp. 337-344.

[3] M. Ainsworth and J.T. Oden, A unified approach to a posteriori error estimation using element residual methods, Numer. Math., 65 (1993), pp. 23-50.

[4] M. Ainsworth and J.T. Oden, A posteriori error estimates for Stokes and Oseen equations, SIAM J. Numer. Anal., 34 (1997), pp. 228-245.

[5] M. Ainsworth and J.T. Oden, A posteriori error estimation in finite element analysis, Comp. Meth. Appl. Mech. Engrg, 142 (1997). pp. 1-\$S.

[6] M. Alexandrov and J.E Dennis, Jr.. R.M. Lewis. and V. Torczon, A trust region framework for managing the use of approrimation models in optimization, in preparation.

[7] I. Babuška and W.C. Rheinboldt, A posteriori error estimates for the finite element method, Int. J. Numeri. Methods Engrg., 12 (1978), pp. 1597-1615.

[8] I. Babuska, T. Strouboulis, C.S. Upadhyay and S.K. Gangaraj, A posteriori and adaptive control of the pollution error in the $h$-version of the finite element method, Int. J. Numer. Methods Engrg. 38 (1995), pp. 4207-4235.

[9] R.E. Bank, Analysis of a local a posteriori error estimate for elliptic equations, in Accuracy Estimates and Adaptive Refinements in Finite Element Computations (eds: I. Babuška, O.C. Zienkiewics, J. Gago, E.R. de A. Oliveira), John Wiley. New York, 1986, pp. $119-128$.

[10] R.E. Bank and A. Weiser, Some a posteriori error estimators for elliptic partial differential equations, Math. Comp., 44:170(1985), pp. 283-301.

[11] R.E. Bank and B.D. Welfert, A Posteriori error estimates for the Stokes problem: a comparison Math. Comput. 82 (1990), pp. 323-340.

[12] R.E. Bank and B.D. Welfert, A posteriori error estimates for the Stokes problem, SIAM J. Numer. Anal., 28 (1991), pp. 591-623.

[13] R. Becker and R. Rannacher, Weighted a posteriori error control in finite element methods, IWR Preprint 96-1 (SFB 359), Heidelberg, 1996.

[14] R. Becker and R. Rannacher, A feedback approach to error control in finite element methods: basic analysis and examples, IWR Preprint 96-52 (SFB 359), Heidelberg, 1996.

[15] F. Brezzi and M. Fortin, Mixed and Hybrid Finite Element Methods, Springer-Verlag, New York, 1991. 
[16] M. Crouzeix and P.-A. Raviart, Conforming and non-conforming finite elements methods for solving the stationary Stokes equation, RAIRO Anal. Numer. 7:33-76, 1973.

[17] P. Ladeveze and D. Leguillon, Error estimation procedures in the finite element method and applications, SIAM J. Numer. Anal., 20 (1983), pp. 485-509.

[18] P. Ladeveze, P. Marin, J.P. Pelle, and Gastine, Accuracy and optimal meshes in finite element computation for nearly incompressible materials, Comp. Meth. Appl. Mech. Engrg., 94:3 (1992), pp. 303-315.

[19] M. Paraschivoiu, and A. T. Patera, A hierarchical duality approach to bounds for the outputs of Partial Differential Equations, Comp. Methods Appl. Mech. Engrg., to appear.

[20] M. Paraschivoiu, J. Peraire, and A. T. Patera, A posteriori finite element bounds for linear-functional outputs of elliptic Partial Differential Equations, Comp. Methods Appl. Mech. Engrg., 150 (1997), pp. 2\$9-312.

[21] M. Paraschivoiu, A posteriori finite Element Bounds for Linear-Functional Outputs of Coercive Partial Differential Equations and of the Stokes problem, Ph.D. Thesis, Department of Mechanical Engineering. M.I.T., October, 1997.

[22] M. Paraschivoiu, J. Peraire, Y. Maday: and A. T. Patera, Fast bounds for outputs of partial differential equations, in Proc. AFOSR Workshop on Optimal Design and Control, Washington, DC, Sept. 30-Oct. 3, 1997.

[23] A.T. Patera and E.M Ronquist, Introduction to Finite Element Methods. Application to Incompressible Fluid Flow and Heat Transfer, 1, in preparation.

[24] J. Peraire and A. T. Patera, Bounds for linear-functional outputs of coercive partial differential equations: Local indicators and adaptive refinement, in Proc. of the Workshop On New Advances in Adaptive Computational Methods in Mechanics, Elserier, Ed. P. Ladeveze and J.T. Oden, Cachan, September, 1997.

[25] J. Peraire and A. T. Patera. Asymptotic a posteriori finite element bounds for the outputs of noncoercive problems: the Helmholtz and Burgers equations, submitted to Comp. Meth. Appl. Mech. Engrg., Dec. 1997.

[26] A. Quarteroni and A. Valli, Numerical Approximation of Partial Differential Equations, Springer-Verlag, Berlin, 1994.

[27] R. Rannacher and F.-T. Suttmeier, A feedback approach to error control in finite element methods: application to linear elasticity. IWR Preprint 96-42 (SFB 359), Heidelberg, 1996.

[28] G. Strang, Introduction to Applied Mathematics, Wellesley-Cambridge Press, Wellesley, Massachusetts, 1986. 
[29] H. Vailong, A Posteriori Bounds for Linear-Functional Outputs of Hyperbolic Partial Differential Equations, S.M. Thesis, Department of Aeronautics and Astronautics, M.I.T., 1997.

[30] R. Verfürth, A posteriori error estimation and adaptive mesh-refinement techniques, J. Comp. Appl. Math., 50 (1994), pp. 67-83.

[31] R. Verfürth, A posteriori error estimators for the Stokes equations, Numer. Math., 55 (1989), pp. 309-325.

[32] S. Yeşilyurt and A.T. Patera, Surrogates for numerical simulations; optimization of eddy-promoters heat exchangers, Comput. Methods Appl. Mech. Engrg 121 (1995), pp. $231-257$. 